\title{
Consistent Hybrid Finite Volume/Element Formulations: Model and Complex Viscoelastic Flows
}

\author{
M.S. Chandio, K.S. Sujatha and M. F. Webster ${ }^{*}$ \\ Institute of non-Newtonian Fluid Mechanics, \\ Department of Computer Science, \\ University of Wales Swansea, \\ Singleton Park, SA2 8PP, \\ Swansea, United Kingdom.
}

\begin{abstract}
The accuracy and consistency of a new cell-vertex hybrid finite element/volume scheme are investigated for viscoelastic flows. Finite element discretisation is employed for the momentum and continuity equation, with FV applied to the constitutive law for stress. Here, the interest is to explore the consequences of utilizing conventional cell-vertex methodology for an Oldroyd-B model and to demonstrate resulting drawbacks in the presence of complex source terms on structured and unstructured grids. Alternative strategies worthy of consideration are presented. It is demonstrated how high-order accuracy may be achieved in steady-state by respecting consistency in the formulation. Both FE and FV spatial discretisations are embedded in the scheme, with FV triangular sub-cells referenced within parent triangular finite elements. Both model and complex flow problems are selected to quantify and assess accuracy, appealing to analysis and experimental validation. The test problem is that of steady sink flow, a pure extensional flow, which reflects some of the numerical difficulties involved in solving more generalised viscoelastic flows, where both source and flux terms may contribute equally. In addition, a complex transient filament-stretching flow is chosen to compute the evolution of stress fields within liquid bridges. Shortcomings of the various upwinding schemes are discussed in this context, whilst dealing with such free-surface type problems. Here, fluctuation distribution alone is advocated and a Lax-scheme is found to deliver accuracy and stability to the computational results, comparing well with the literature.
\end{abstract}

\section{Introduction}

Finite volume (FV) high-resolution upwind methods for hyperbolic conservation laws and their extensions have been the subject of intensive research for more than half a century, with remarkable advances in the last two decades or so [2-13]. These methods have now reached a stage of maturity and play a central role in numerical discretisation of differential equation systems. The FV approach may be viewed as a "method of moments" FE formulation, with weighting functions taken as unity. Literature of relevance here falls into two categories, those with complete FV implementations [3] and those of hybrid form [2,10,12,13]. Early numerical simulations for viscoelastic fluids were performed using FE Galerkin discretisation in conjunction with upwinding techniques (Streamlineunwinding, SU, streamline upwinding Petrov-Galerkin, SUPG) [1,11]. In the FV domain, considerable progress has been made for pure advection equations $[4,9]$ and has sufficiently advanced to accommodate viscoelastic flows, where source terms may play a dominant role.

\footnotetext{
*Author for correspondence email: m.f.webster@swan.ac.uk
} 
The contributions of Crumpton et al. [6], Struijs et al. [9] are worthy of mention. These works have implemented cell-vertex FV formulations for compressible Navier-Stokes, Euler and advection equations, respectively. On triangles, Berzins and Ware [8] concentrated on a cell-centered FV approach. The distinction between cell-centred and cell-vertex schemes lies in the level of auxiliary interpolation and the upwinding techniques appropriate for each. In cell-centred methods, solution variables are positioned at the centroids of the control volume. In contrast, cell-vertex methods use the mesh points to locate solution variables.

Sato and Richardson [10] employed a time-explicit FE-method on the momentum equation and time-implicit FV-method for pressure and stress, of cell-centred type. In order to achieve high-order upwinding, a total variational diminishing flux corrected transport (TVD-FCT) schemes was applied to the advection terms of the constitutive equation. Darwish and Whiteman [3] implemented a staggered grid-FV method, and Crumpton et al. [6] developed a cell-vertex FV method on structured quadrilateral and hexahedral meshes for Navier-Stokes equations.

In the present study, our attention is focused mainly on the contrast between consistent and inconsistent treatments for source and flux terms within cell-vertex finite volume formulations. A model sink flow problem is chosen to quantify accuracy and consistency, addressing both structured and unstructured meshing. Once, this is established, the proposed hybrid FE/FV method is employed on a complex viscoelastic flow with free-surface. The drawbacks of various FV implementations are discussed. Here, specifically we address filament-stretching for viscoelastic fluids, focusing upon constant shear-viscosity fluid properties. It is appropriate to consider fluid representation through the Oldroyd-B model, for which we adopt a high polymeric viscosity contribution. Here, we discuss the break-up of viscoelastic liquid-bridges under elongation, where the stretching of the liquid-bridge is confined between two end-plates. Such a setting may be used in rheometry to measure the elongational viscosity of a fluid [18]. Over the preceding decade, much interest has been expressed in the area of extensional rheology for polymer solutions [19-22]. In this regard, the filament-stretching rheometer, introduced by Tritaatmadja and Sridhar [18], has proved to be a useful experimental tool. The primary goal of these studies has been to determine the nature of deformation in the central portion of the filament. This detects if the flow approaches ideal uniaxial elongation and leads to the observation of rheological properties, that either enhance or restrain ideal uniaxial deformation.

Finite element simulations for viscous and viscoelastic fluids within filament-stretching flows has been performed extensively in the literature, see [19-22]. A detailed literature review on this problem is presented in our precursor work [24]. In this work, we propose the appropriate finite volume scheme to be employed to model such complex flow problems. We illustrate the differences between fluctuation distribution and median-dual-cell constructs, within a cell-vertex discretisation on structured and unstructured triangular meshes. Also, various fluctuation distribution schemes, namely, LDB, PSI and Lax-Wendroff, are contrasted in this study.

\section{Governing equations}

The governing equations for incompressible, viscoelastic flows are represented by conservation laws for mass and momentum, in conjunction with an equation of state for stress. Posed in nondimensional form, the balance equations under isothermal flow conditions may be expressed as

$$
\square . \mathbf{u}=0
$$

$$
\operatorname{Re} \frac{\partial \mathbf{u}}{\partial \mathrm{t}}=\square \operatorname{Re} \mathbf{u} \cdot \square \mathbf{u} \square \square \mathrm{p}+\square \cdot\left(2 \frac{\square_{\mathrm{s}}}{\square_{0}} \mathbf{d}+\square\right)
$$


and the constitutive equation for an Oldroyd-B fluid may be expressed as

$$
\mathrm{We} \frac{\partial \square}{\partial \mathrm{t}}=\square \mathrm{Weu} \cdot \mathrm{CDO} \square+2 \frac{\square_{\mathrm{e}}}{\square_{0}} \mathbf{d}+\mathrm{We}\left(\mathbf{L} \cdot \square+\square \mathbf{L}^{\dagger}\right),
$$

where $\mathbf{u}, \mathrm{p}, \sqcup$ represent the fluid velocity, the hydrodynamic pressure and the extra-stress tensor. The total viscosity $\square_{0}$ is split into solvent $\left(\square_{\mathrm{s}}\right)$ and polymeric $\left(\square_{\mathrm{e}}\right)$ contributions, such that $\square_{0}=\square_{\mathrm{s}}+\square_{\mathrm{e}}$; $\mathbf{d}=\left(\mathbf{L}+\mathbf{L}^{\dagger}\right) / 2$ represents the Euler rate-of-deformation tensor and $\quad \mathbf{L}^{\dagger}=\square . \mathbf{u}$, the velocity gradient. The non-diamentional variables, namely, velocity, pressure, length, stress and time may be expressed as

$$
\mathrm{u}^{*}=\frac{\mathrm{u}}{\mathrm{U}}, \mathrm{p}^{*}=\frac{\mathrm{L}}{\square_{0} \mathrm{U}}, \mathrm{x}^{*}=\frac{\mathrm{x}}{\mathrm{L}}, \square^{*}=\frac{\mathrm{L}}{\square_{0} \mathrm{U}} \square \square^{*}=\frac{\square}{\square_{0}}, \mathrm{t}^{*}=\mathrm{t} \frac{\mathrm{L}}{\mathrm{U}}
$$

The Reynolds and Weissenberg numbers are defined as

$$
\mathrm{Re}=\frac{\square \mathrm{UL}}{\square_{0}}, \quad \mathrm{We}=\frac{\square \mathrm{U}}{\mathrm{L}}
$$

where $\square, \square$ are the fluid density and relaxation time and $\mathrm{U}, \mathrm{L}$ are characteristic velocity and length scale of the flow. The zero shear-rate viscosity (constant)is taken as the characteristic scale for viscosity.

\section{Numerical algorithm}

We outline the structure of the FE scheme, and point to the hybrid FE/FV options in contrast, referring to Matallah et al. [1] and Wapperom and Webster [2] for detailed discussion. The general framework is a time-splitting semi-implicit formulation, involving two distinct aspects: a TaylorGalerkin scheme and a pressure-correction scheme. The Taylor-Galerkin scheme is a two-step time stepping procedure, extracted via a Taylor series expansion in time[45]. The pressure-correction method accommodates the incompressibility constraint to ensure second-order accuracy in time. A three-stage structure emerges per time step,that may be expresses in discrete form as [2]:

Stage 1a: $\quad \frac{2}{\square \mathrm{t}} \mathrm{A}_{\mathrm{u}}\left(\mathrm{U}^{\mathrm{n}+\frac{1}{2}} \square \mathrm{U}^{\mathrm{n}}\right)=\mathrm{b}_{\mathrm{u}}\left(\mathrm{p}^{\mathrm{n}}, \mathrm{U}^{\mathrm{n}}, \mathrm{T}^{\mathrm{n}}, \mathrm{D}^{\mathrm{n}}\right)$,

$$
\frac{2 \mathrm{We}}{\square \mathrm{t}} \mathrm{A}_{\square}\left(\mathrm{T}^{\mathrm{n}+\frac{1}{2}} \square \mathrm{T}^{\mathrm{n}}\right)=\mathrm{b}_{\square}\left(\mathrm{U}^{\mathrm{n}}, \mathrm{T}^{\mathrm{n}}, \mathrm{D}^{\mathrm{n}}\right)
$$

Stage 1b: $\quad \frac{A_{u}}{\square t}\left(U^{*} \square U^{n}\right)=b_{u}\left(p^{n}, U^{n}, U^{n+\frac{1}{2}}, T^{n+\frac{1}{2}}, D^{n+\frac{1}{2}}\right)$,

$$
\frac{\mathrm{We}}{\square \mathrm{t}} \mathrm{A}_{\square}\left(\mathrm{T}^{\mathrm{n}+1} \square \mathrm{T}^{\mathrm{n}}\right)=\mathrm{b}_{\square}\left(\mathrm{U}^{\mathrm{n}+\frac{1}{2}}, \mathrm{~T}^{\mathrm{n}+\frac{1}{2}}, \mathrm{D}^{\mathrm{n}+\frac{1}{2}}\right)
$$

stage 2: $\quad \frac{\square t}{2} A_{2}\left(p^{n+1} \square p^{n}\right)=b_{2}\left(U^{*}\right)$, 
Stage 3: $\quad \frac{2}{\square \mathrm{t}} \mathrm{A}_{3}\left(\mathrm{U}^{\mathrm{n}+1} \square \mathrm{U}^{*}\right)=\mathrm{b}_{3}\left(\mathrm{p}^{\mathrm{n}}, \mathrm{p}^{\mathrm{n}+1}\right)$

Here the notation implies, superscript $n$ denoting the time level, $\square \mathrm{t}$ the time step and U, U ${ }^{*}, \mathrm{P}, \mathrm{T}, \mathrm{D}$ the nodal values of velocity, non-solenoidal velocity, pressure, extra-stress and velocity gradient respectively. $A_{U}, A_{2}, A_{3}$ are the standard velocity, stiffness and mass matrices. The precise form of $\mathrm{A}_{\square}$ varies according to the implementation, either FE or hybrid FE/FV. Galerkin spatial FE

discretisation is employed within the momentum equation in stage 1, the pressure-correction in stage 2 and the incompressibility constraint in stage 3. The diffusive terms in Eq. (3) are treated in a semiimplicit manner in order to enhance stability. The resulting Galerkin mass matrix-vector equations at stages 1 and 3 are solved using an element-by-element Jacobi scheme. The required levels of convergence are attained with only a few Jacobi iterations. A direct Choleski decomposition procedure is invoked to handle stage 2 .

The finite volume approach adopted in this work, and applied to stress alone, is of cell-vertex orientation. Cell-vertex schemes, generally maintain their accuracy for broader families of unstructured meshes [15]. The core of these cell-vertex FV schemes is associated with the dictates of fluctuation distribution (FD), governed by FD-coefficients $\left\{\square_{l}^{T}\right\}_{l=1,3}$ on triangular control-volume T ; see [4]. These schemes are compact-stencil upwinding techniques. More detail, on the theory and construction of such upwinding schemes, can be found in Hubbard et al. [4]. They require only the vertices of a triangular cell to evaluate the solution of a given scalar field. The FD-schemes differ based on the appropriate discretisation choices for pure advection equation, and upon properties such as conservation, linearity preservation and positivity.

\subsection{Fluctuation distribution schemes}

Fluctuation distribution schemes can be categorised into those of linear and non-linear types. Both linear and non-linear upwind distribution schemes on unstructured triangular meshes have been developed over the past years, with built-in properties such as positivity $(\mathrm{P})$ and linearity preservation (LP). Linear schemes can be either P or LP. For both properties to hold, the scheme must be of nonlinear form.

$L D B$ scheme: This is a linearity-preserving (non-positive) scheme defined on each triangle by the angle $\square^{\dagger}$ subtended at an inflow vertex by the advection velocity $\boldsymbol{a}$ (an average vector per cell). The LDB distribution coefficients $\square_{l}^{\mathrm{T}}$, defined per cell node $i$, may be expressed as:

$$
\begin{aligned}
& \square_{\mathrm{i}}=\left(\sin \square_{1} \cos \square_{2}\right) / \sin \left(\square_{1}+\square_{2}\right), \\
& \square_{\mathrm{j}}=\left(\sin \square_{2} \cos \square_{1}\right) / \sin \left(\square_{1}+\square_{2}\right), \\
& \square_{\mathrm{k}}=0 .
\end{aligned}
$$

We point out that the closer the advection velocity $\boldsymbol{a}$ is to being parallel to one of the cell boundaries, the larger the contribution to the downstream node at that boundary.

PSI scheme: This scheme is non-linear and possesses both positive and linearity preserving characteristics. It is equivalent to the $\mathrm{N}$-scheme with a minmod limiter [9]. The $\mathrm{N}$-scheme is a linear

\footnotetext{
${ }^{\dagger}$ The equivalent area-weighted version is called LDA-scheme
} 
$\square$-scheme, which is positive. It is optimal in the sense that it may use the maximum allowable timestep and the narrowest stencil. The resulting $\square_{i}$ coefficients of the $\mathrm{N}$ scheme, for the case of two inflow sides are,

$$
\begin{aligned}
& \square_{\mathrm{i}}=\square \mathrm{k}_{\mathrm{i}}\left(\square_{\mathrm{i}} \square \square_{\mathrm{k}}\right), \\
& \square_{\mathrm{j}}=\square \mathrm{k}_{\mathrm{j}}\left(\square_{\mathrm{i}} \square \square_{\mathrm{k}}\right), \\
& \square_{\mathrm{k}}=0 .
\end{aligned}
$$

where, coefficients k may be expressed as:

$$
\mathrm{k}_{l}=\frac{1}{2} \mathbf{a} \cdot \mathbf{n}_{l}, \quad \prod_{l=1}^{3} \mathbf{n}_{l}=0, \quad \prod_{l=1}^{3} \mathrm{k}_{l}=0
$$

If we denote the coefficients of the PSI scheme by $\square_{l}^{*}$ and of the $\mathrm{N}$-scheme by $\square$, as in equation (8), the PSI scheme only deviates from the N-scheme if $\square_{i} \square_{j}<0$, and we have

$$
\begin{aligned}
& \square_{i}^{*}=\square_{\mathrm{i}} \square \mathrm{L}\left(\square_{\mathrm{i}}, \square \square_{\mathrm{j}}\right), \\
& \square_{j}^{*}=\square_{\mathrm{j}} \square \mathrm{L}\left(\square_{\mathrm{j}}, \square \square_{\mathrm{j}}\right) .
\end{aligned}
$$

Here, $\mathrm{L}(\mathrm{x}, \mathrm{y})$ is the minmod limiter function, defined via the conventional 'sign' operator,

$$
\mathrm{L}(\mathrm{x}, \mathrm{y})=\frac{1}{4}(1+\operatorname{sign}(\mathrm{xy}))(\operatorname{sign}(\mathrm{x})+\operatorname{sign}(\mathrm{y})) \min (|\mathrm{x}|,|\mathrm{y}|) \text {. }
$$

Lax-Wendroff (Lax) scheme: This scheme is second-order and linear, satisfying linearitypreservation. It is spatially-centered and second-order accurate in space. In addition, it contains a dissipation term designed to control oscillations in the neighbourhood of discontinuities, thus conferring second-order accuracy in time also. For the Lax-scheme, the distribution coefficients $\square_{l}^{\mathrm{T}}$ may be expressed as:

$$
\square_{l}^{\mathrm{T}}=\frac{1}{3}+\frac{\square \mathrm{t}}{4 \square_{\mathrm{T}}} \mathbf{a} \cdot \mathbf{n}_{l}^{\mathrm{T}}
$$

where $\square \mathrm{t}$ is a time-step size, $\mathbf{a}$ is an averaged advection velocity and $\mathrm{n}_{l}^{\mathrm{T}}$ is a scaled inward-pointing normal vector to an edge of triangle T, opposing node $l$.

\section{The hybrid finite element / finite volume method}

The finite element grid is used as a platform for the finite volume grid, from which control volumes are constructed. Each FV cell is a sub-triangle resulting from a parent FE cell, see Fig. 1a. The unique subtended median-dual-cell zone $(m d c)$, around a particular node $l$, surrounded by FV sub-cells T, is illustrated in Fig.1b. The six-noded parent FE cell is based on a triangular cell with three vertices and three mid-side nodes. Velocity interpolation is achieved via quadratic shape functions, using the six nodal values, and the pressure by linear functions based upon the vertices alone. The stresses are computed on the vertices of the FV cells as outlined below.

First, we recast the stress constitutive equation (3) in conservative form,

$$
\frac{\partial \square}{\partial \mathrm{t}}+\mathrm{\square} \cdot \mathrm{R}=\mathrm{Q}
$$


where the flux $\mathbf{R}$ and source $\mathbf{Q}$ can be defined as:

$$
\begin{gathered}
\mathbf{R}=\mathbf{u} \square \\
\mathbf{Q}=\frac{1}{\mathrm{We}}\left(2 \frac{\square_{\mathbf{e}}}{\square_{0}} \mathbf{d} \square+\left(\mathbf{L} \cdot \square+\square \mathbf{L}^{\dagger}\right) .\right.
\end{gathered}
$$

Velocity gradients are represented via recovery procedures [1]. It is common practice to refer to a single scalar component $\square$ of stress, with correspondence in flux and source terms. In the presence of source terms, the standard treatment advocate widely in the literature consists in dealing separately with the flux and source terms (inconsistent approach). Here, in contrast, a new formulation is proposed, whereby both flux and source terms are evaluated in a consistent manner.

\subsection{Inconsistent fv-nodal update}

Here, the fluxes are distributed using $\square_{l}$ FD coefficients on the whole FV cell area, whereas the control volume for the source term, the $m d c$, is one-third that of the FV cell area. Hence, this formulation is inconsistent, both in respect of control volume usage and reference to $\square_{l} \mathrm{FD}$ coefficients.

$$
\square_{l} \frac{\square_{l}^{\mathrm{n}+1} \square \square_{l}^{\mathrm{n}}}{\square \mathrm{t}}=\square_{l}^{\mathrm{T}} \mathrm{R}_{\mathrm{T}}+\mathrm{Q}_{\mathrm{mdc}},
$$

where $\mathrm{R}_{\mathrm{T}}$ is the integral of the flux (R) over triangle $\mathrm{T}$ (see Eq $(24,25)$ ), and $\mathrm{Q}_{m d c}$ is the source associated with node $l$ taken over the area of the surrounding $m d c,\left(\mathrm{Q}_{\mathrm{mdc}}=\square \mathrm{d} \square \mathrm{mdc}\right)$, respectively.

\subsection{Consistent fv-nodal update}

Here, both fluxes are sources are distributed alike using $\square_{l}$ FD coefficients on the whole FV cell area,

$$
\square_{l} \frac{\square^{\mathrm{n}+1} \square \square_{l}^{\mathrm{n}}}{\square \mathrm{t}}=\square_{\square \mathrm{T}}^{\mathrm{T}}\left(\mathrm{R}_{\mathrm{T}}+\mathrm{Q}_{\mathrm{T}}\right) .
$$

The notation $\square_{\square \mathrm{T}}$, represents summation over contribution from all FV-cells $(\mathrm{T})$ surrounding node $\quad l$, and $\square_{l}$ the area of the median dual cell $(\quad m d c)$ associated with node $l$. In addition, we have FDfactors $\square_{l}^{\mathrm{T}}$, temporal stress $\square^{\mathrm{T}}$ at node $l$, and \{flux $\mathrm{R}_{\mathrm{T}}$, source $\mathrm{Q}_{\mathrm{T}}$ \} contributions on FV-triangle, $\mathrm{T}$ $\left(Q_{\mathrm{T}}=\underset{\square_{\mathrm{T}}}{\square d} \square_{\mathrm{T}}\right)$. Here, both LDB and PSI schemes are used for the evaluation of $\quad \square_{l}^{\mathrm{T}}$-FD coefficients. The scheme following stencil (17) is consistent both in $\square_{l}^{\mathrm{T}}$-FD coefficient application and control volume.

\section{Hybrid combinations}


Results are obtained by switching flux and source term treatment between LDB and PSI schemes, to determine the influence of positivity upon the computations, and in particular its consistent application to flux terms. This is achieved through stencils of:

$$
\begin{aligned}
& \square \\
& \square_{l} \frac{\square^{\mathrm{n}+1} \square \square_{l}^{\mathrm{n}}}{\square \mathrm{t}}=\square_{l(l d b)}^{\mathrm{T}} \mathrm{R}_{\mathrm{T}}+\square_{l(p s i)}^{\mathrm{T}} \mathrm{Q}_{\mathrm{T}}, \\
& \square_{l} \frac{\square_{l}^{\mathrm{n}+1} \square\left[\square_{l}^{\mathrm{n}}\right.}{\square \mathrm{t}}=\square_{l(p s i)}^{\mathrm{T}} \mathrm{R}_{\mathrm{T}}+\square_{l(l d b)}^{\mathrm{T}} \mathrm{Q}_{\mathrm{T}} .
\end{aligned}
$$

\section{Median dual cell considerations}

In order to retain stability at high We numbers, Aboubacar et al [12] and Webster et al13], improved the consistent approach (17) by appealing to median dual cell contributions. Summing over all $f v$-subcells surrounding node $l$ yields the $\mathrm{CT}_{3}$-scheme, provides a generalized stencil:

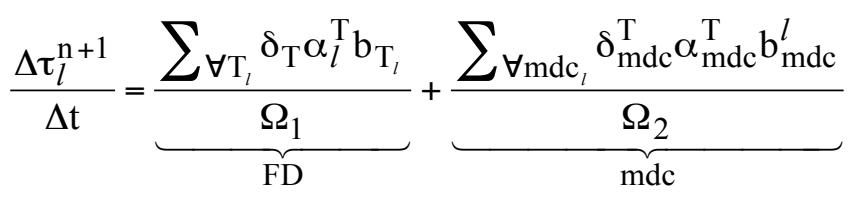

where $\mathrm{b}_{\mathrm{T}_{l}}=\left(\mathrm{R}_{\mathrm{T}}+\mathrm{Q}_{\mathrm{T}}\right), \quad \mathrm{b}_{\mathrm{mdc}}^{l}=\left(\mathrm{R}_{\mathrm{mdc}}+\mathrm{Q}_{\mathrm{mdc}}\right)^{l}$, and appealing to $\square_{\mathrm{FD}}=\square_{\mathrm{T}_{l}} \square_{\mathrm{T}} \square_{l}^{\mathrm{T}} \square_{\mathrm{T}_{l}}$, and $\square_{\mathrm{mdc}}=\square \mathrm{mdc}_{l}, \square_{\mathrm{mdc}}^{\mathrm{T}} \square_{l}^{\mathrm{T}_{l}}$. Theoretically, $\square_{\mathrm{T}}$ and $\square_{\mathrm{mdc}}$ are mutually linked and complementarily exclusive. Blending FD and $m d c$ contributions with parameters $\square_{\mathrm{T}}$ and $\square_{\mathrm{mdc}}$ provides various alternative scheme combinations $\left(\mathrm{CT}_{0}, \mathrm{CT}_{2}, \mathrm{CT}_{3}\right.$ see $\left.[12,13]\right)$. These may be categorized in a generalized manner, around area weighting factors $\square_{1}$ and $\square_{2}$, and blending parameters, $\square_{\mathrm{T}}$, and $\square_{\mathrm{mdc}}$. We obtain the $\mathrm{CT}{ }_{3}$-scheme by setting $\square_{1}=\square_{2} \equiv \square_{\mathrm{FD}}+\square \mathrm{mdc}$; alternatively, we can recover the $\mathrm{CT}_{2}$-scheme with the combination $\square_{1}=\square_{\mathrm{FD}}$ and $\square_{2}=\square_{\mathrm{mdc}}$; the original nodal-update $\left(\mathrm{CT}{ }_{0}\right)$ corresponds to $\square_{1}=\square_{2} \equiv \hat{\square}_{l}$.

Fluctuation distribution coefficients $\square_{l}^{\mathrm{T}}$, may be provided via an appropriate choice of scheme, for example, LDB or PSI. Both such options offer second-order accuracy at steady-state. Wapperom and Webster [2], recommended setting of blending factors as $\square_{\mathrm{T}}=\square$ and $\square_{\mathrm{mdc}}=1$, with $\square=\square / 3$ if $|\square \square 3|$ and 1 otherwise. Here, $\square=W e(\mathbf{a} / \mathrm{h})$, with a the magnitude of an advection velocity per $\mathrm{FV}$-cell and $\mathrm{h}$ the square-root of the area of the FV-cell in question. With the above parameter combinations ( $\mathrm{so}_{\mathrm{mdc}}=1$ ), both $\mathrm{CT} \quad{ }_{2}$ and $\mathrm{CT} \quad{ }_{0}$ variants proved inadequate in tracking transient solution evolution in a start-up planar Poiseuille flow. In particular, the $\mathrm{CT}_{2}$-scheme was sensitive to the setting of $\square_{\mathrm{mdc}}$. There, significant improvement in transient accuracy was achieved with the CT $2^{-}$ scheme by appealing to a dynamic $\square_{\mathrm{mdc}}$ factor setting in the form, $\square_{\mathrm{mdc}}=1 \square \square_{\mathrm{T}}$ In contrast, the $\mathrm{CT}_{3^{-}}$ scheme was relatively insensitive to $\square_{\mathrm{mdc}}$ setting, as it encompasses consistent treatment of controlvolumes for flux and source terms on the $r h s$, and consistent area-weighting for time-terms on the $l h s$ of the equation. Hence, this is the preferred option of choice.

In the current work, we have studied two different viscoelastic flow problems. A model steady problem of sink flow is chosen to analyse consistency and accuracy of the proposed scheme 
alternatives. In addition, to validate accuracy under more severe settings, a complex problem is investigated, that of transient free-surface filament stretching flow. Here, temporal as well as spatial issues must be resolved.

\section{Sink flow problem}

A steady sink flow problem [16] is solved in two-dimensions in the r-z plane for an Oldroyd$B$ fluid. This represents radial sink flow, from the outer radius $r=R_{0}$ to the inner radius $r=R_{i}$, with radial velocity component given by $\mathbf{U}_{\mathbf{r}}=\square \frac{\mathbf{k}}{\mathbf{r}}$ and velocity gradient $\frac{\partial \mathbf{U}_{\mathbf{r}}}{\partial \mathbf{r}}=\frac{\mathbf{k}}{\mathbf{r}^{2}}$, for constant $\mathrm{k}$ (see

Fig. 2). The dominant stress component $\square_{\mathrm{r}}$ in this flow is determined numerically on the basis of frozen kinematics. We adopt a Weissenberg number $W e=0.1$, and polymeric to total-viscosity ratio as $\frac{\square_{\mathbf{e}}}{\square_{0}}=\frac{8}{9}$. The results obtained are compared with an analytic solution (see Gunter [16]):

$$
\square_{\mathrm{rr}}=\frac{\square_{\mathrm{e}}}{\square \mathrm{y}}\left(1+\mathrm{C}_{\mathrm{r}} \mathrm{e}^{\mathrm{y}}\right)
$$

where

$$
\begin{aligned}
& \mathrm{y}=\frac{\mathrm{r}^{2}}{2 \mathrm{k} \square}, \\
& \mathrm{C}_{\mathrm{r}}=\left(\square_{\mathrm{rr}} \frac{\square \mathrm{Y}_{0}}{\square_{\mathrm{e}}} \square 1\right) \mathrm{e}^{\square \mathrm{y}_{0}},
\end{aligned}
$$

Here, $\square$ is the prescribed stress at the outer boundary $R_{0}$ and $Y_{0}=y\left(R_{0}\right)$.

For this model problem, consistency of FV-treatment across source and flux terms is foremost. Calculations are performed on triangular elements on both structured and unstructured meshes in order to investigate situations with different inflow sides. Through mesh refinement and calibration against an analytical solution, orders of accuracy are established for the various FV-schemes outlined above.

The parameters chosen for this flow problem are: outer radius $\mathrm{R}_{0}=2$, inner radius $\mathrm{R}_{\mathrm{i}}=1$ and $\mathrm{k}=2$, implying an inlet velocity $\mathrm{U}_{r}=-1$ at $\mathrm{R}_{0}$ and outlet velocity $\mathrm{U}_{r}=-2$ at $R_{\mathrm{i}}$. The axial coordinates are taken as $Z_{0}=1$ and $Z_{l}=2$. Limiting tolerance to detect convergence is taken as $10^{-8}$. The time-step used, is $\square \mathrm{t}=0.01$ for $5 \times 5,10 \times 10$ and $10 \times 5$ meshes, $\square \mathrm{t}=0.005$ for $20 \times 5$ and $20 \times 20$, and for $40 \times 5$ and $40 \times 10$ meshes $\square \mathrm{t}=0.0025$. The infinity norm $\quad \square \square=\left\|\square^{\mathrm{h}} \square L_{\text {exact }}\right\|$, scaled by a maximum value of the exact solution, is reported for comparison of solutions. To reduce some of the degrees of complexity in the problem, we have chosen to freeze velocity (with analytic gradient) and pressure fields (linearisation/localisation), so that unequivocally, we may concentrate on accuracy of FV-stress representation (see also, Wapperom and Webster [2]). Boundary conditions for the stress are specified on the inflow boundaries $\mathrm{r}=\mathrm{R}_{0}$. For the time-stepping procedure initial conditions are taken as a quiescent state, by default. We note that, $\square\left(R_{0}\right)$ may be set assuming fully-relaxed stress at infinite distance from the sink. 


\subsection{Results for sink flow}

Within the tables of results, we chart the relative difference between analytic and numerical solution, in the infinity norm, on the principle stress component $\square_{r}$, over a range of meshes. In this study, the resultant $R_{T}$ is evaluated in two different forms, either as a boundary integral around $\square_{T}$, as standard,

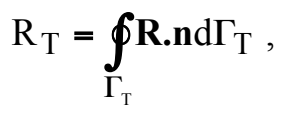

or, as an area integral,

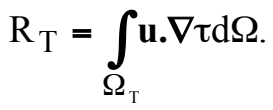

These results demonstrate the trends with mesh refinement of the various scheme choices. This provides insight into accuracy attainment for each scheme, that reflects, their spatial convergence rates, reported in terms of $\mathrm{O}\left(\mathrm{h}^{\mathrm{p}}\right)$. We take results in order of structured meshes (see Fig.3), prior to unstructured (see Fig.4). In each instance, we can explore the influence that consistency of implementation has on accuracy. Here, scheme-S1, represents a cell-boundary integral approach, scheme-S2, boundary integral with fixed stress on the boundaries, and scheme-S3, an area integral procedure.

\section{Structured mesh solutions}

In Fig.5 (a-b) and Table 1, we compare the consistent (Eq. (17)) and inconsistent ( Eq. (16)) FV formulations. With this set of results, there is only one-inflow side per FV-cell, so that only one node of a triangle will receive an update. This employs therefore a simplistic upwinding treatment. First, we consider scheme-S1, with boundary integral approximation for flux terms. Convergence rates for the consistent implementation are $\mathrm{O}\left(\mathrm{h}^{1.5}\right)$ on average, with finer-mesh local-rates tending to $\mathrm{p}=1.1$. The inconsistent counterpart (reflecting the standard version frequently cited in the literature), fairs only slightly less well on average, $\mathrm{O}\left(\mathrm{h}^{1.2}\right)$, and closely matches findings on finer meshes. So here, there is not much to choose between these two options, both being around first-order in accuracy. The level of error itself is significantly larger with the inconsistent case: by as mush as a factor of four on the finest mesh.

If a uniform fluctuation distribution is adopted, that countermands upwinding with $\square_{1}=1 / 3$, then similar findings emerge. Here, it is apparent that an $m d c$ treatment of sources is equivalent to a consistent fluctuation distribution approach with uniform coefficients. This is the key to appreciate that accuracy is influenced by other factors than the distribution coefficients alone. Generally, with structured meshes, it is observed that through fluctuation distribution, interior nodes acquire contributions from an even number of triangles; alternatively, boundary nodes are updated from a single triangle. Therefore, we include results based upon a fixed-stress boundary condition setting, scheme-S2. With the consistent implementation, we are now able to attain second-order accuracy with mesh refinement, and almost third-order on average. It is apparent, one is losing one order of accuracy here due to the boundary condition issue and lack of balance in the nodal updates with uniform meshing. For the inconsistent counterpart of scheme-S2, first-order accuracy is attained. This is due solely to the inconsistency of treatment between flux (FD) and source terms (on $m d c$ ).

Another aspect that may have a bearing on accuracy is the discrete evaluation of flux, in boundary integral form, and source terms, by area integration. From empirical evidence, it would appear that a close approximation to flux values is gathered at mid-side nodes, whilst sources are well represented at centroid locations. This has lead to the testing of scheme-S3. There instead, fluxes are taken as area integrals, a non-conservative representation of the convected term. In consistent form, 
the average estimate error slope of almost $\mathrm{O}\left(\mathrm{h}^{3}\right)$ is extracted, with mesh refinement, see Fig.5(a-b). Clearly, this equates to optimal scheme choice. Fixing stress boundary conditions could well improve matters further. However, for general problems, this may not always be plausible. Notably, oncemore the inconsistent counterpart to scheme-S3, is restricted to only first-order accuracy: it is the inconsistency that is responsible for this restriction.

\section{Unstructured mesh solutions}

On unstructured meshes and for this model problem, there may be more than one inflow side. Hence, the fluctuation distribution coefficients will vary and nodal updates, by upwinding design, will be distributed to more than one node of each triangle. In this context, it is meaningful to contrast different fluctuation distribution schemes. In addition, mesh areas will differ from one triangle to another and the number of triangles sharing a node will vary. Hence, nodes throughout the mesh will not be updated in a uniform manner. The results for unstructured meshes are presented in Fig.5(c-d) and Table 2.

The main observation that is prominent with unstructured meshes is, there is an order of accuracy lost, in shifting from consistent to inconsistent implementations. Hence, consistency is a dominant issue. Otherwise, there is little to choose between the schemes on average convergence performance, being around $\mathrm{O}\left(\mathrm{h}^{1.7}\right)$ for consistent and $\mathrm{O}\left(\mathrm{h}^{0.7}\right)$ for inconsistent cases. The exception to the rule is the inconsistent area integral flux evaluation of scheme-S3, that loses h-convergence consistency altogether. Notably, consistent schemes, S1 and S3, both tend to around second-order accuracy with mesh refinement. Also, the influence of upwinding in the departure from structured meshes, has most impact upon consistent scheme S1. Here, this is the only instance where there is improvement in order of accuracy with mesh refinement, from $\mathrm{O}\left(\mathrm{h}^{1.1}\right)$ for structured, to $\mathrm{O}\left(\mathrm{h}^{1.8}\right)$ on unstructured meshes. Interestingly, a choice of uniform fluctuation distribution coefficients with $\square_{l}=1 / 3$, gives almost identical convergence properties to scheme $\mathrm{S} 1$. This leads one to appreciate that it is the distribution to more than one node that is the critical factor here.

In Table 3, the point of attention shifts to the treatment of flux and source terms in respect of fluctuation-distribution schemes. Switching the consistent formulation (17) from LDB scheme to PSI, we observed an identical error norm slope, to that obtained for the LDB consistent scheme, see Fig.6(a-b). Further, for the evaluation of $\square_{l}$ FD coefficient, we used the PSI scheme for the flux term and LDB scheme for the source term (Eq. (19)). The convergence rate is improved slightly with mesh refinement; see Table 4. This improvement in convergence rate is due to the suitability of the particular scheme chosen for each term. The inherited properties of positivity and linearity preserving from the PSI scheme, and linearity preserving property of the LDB scheme, together retain solution smoothness in temporal (iterative) convergence to steady-state. They do not generate oscillations in the solution, on either coarse or refined meshes, see Fig.7(a-d). In contrast, when flux terms are computed with the LDB scheme and source terms with the PSI (positive) scheme (Eq.(18)), Fig.6c reflects a negative error slope for all schemes, S1-S3, (see Table 4). This is in keeping with expectation, due to the positivity of the PSI scheme, which may introduce fresh extrema in the solution, on both coarse and refined meshes (see again, Fig. 7 (a-d)). Here, positivity imposed on source terms, under FD-approximation degrades solution accuracy.

\section{Filament stretching flow}

In this second section of the study, we consider a complex transient flow with free-surfaces, that described by the extensional deformation of a viscoelastic filament between two coaxial discs. The bottom plate is held fixed and the top-plate is driven in time at an exponential rate providing a constant extension-rate at the filament centre, see Fig.8. It is assumed that inertia and gravity are 
negligible. The top moving-plate may be set into motion at an exponential rate, so that the dynamic filament-length, $\mathrm{L}_{\mathrm{P}}(\mathrm{t})$, may be represented via

$$
\begin{gathered}
\mathrm{L}_{\mathrm{p}}(\mathrm{t})=\mathrm{L}_{0} \mathrm{e}^{\dot{\mathrm{D}_{0} \mathrm{t}}} \\
\frac{\mathrm{dL}_{\mathrm{p}}(\mathrm{t})}{\mathrm{dt}}=\mathrm{L}_{0} \dot{\square}_{0} \mathrm{e}^{\dot{\mathrm{L}}_{\mathrm{t}} \mathrm{t}}
\end{gathered}
$$

where $\dot{\square}_{0}$ is an imposed initial stretch-rate and $\mathrm{L} \quad{ }_{0}$ is the initial length of the filament. The initial aspect ratio of the liquid-bridge is defined as $\square_{0}=\frac{\mathrm{L}_{0}}{\mathrm{R}_{0}}$, where $\mathrm{R}_{0}$ is the initial radius Here, we have selected $\square_{0}=\frac{1}{3}$. During elongation, the transient aspect ratio of the liquid bridge may be expressed as $\square_{0}=\frac{\mathrm{L}_{\mathrm{p}}(\mathrm{t})}{\mathrm{R}_{0}}$. Material parameters are given in the form: $\square\left(\right.$ density) $=890\left(\mathrm{~kg} \mathrm{~m}^{-3}\right)$, $\dot{\bigsqcup}_{0}$ (stretchrate $)=1.6\left(\mathrm{~s}^{-1}\right), \mathrm{L}_{0}$ (initial length) $=1.0 \times 10^{-3}(\mathrm{~m}), \mathrm{R}_{0}$ (initial radius) $=3.0 \times 10^{-3}(\mathrm{~m}), \square$ (surface tension coefficient $)=28.910^{-3}\left(\mathrm{Nm}^{-1}\right), \square_{0}$ (zero shear viscosity $)=98 \mathrm{~Pa} \mathrm{~s}$.

A rectangular mesh of 20x100 elements, with 8025 nodes and 4000 triangular elements is used to perform the numerical simulation. We choose to scale, length and time, with initial filament length $\mathrm{L}_{0}$ and initial stretch-rate $\frac{1}{\dot{C}_{0}}$, respectively. Stress and pressure are scaled by $\quad \square_{0} \dot{\bigsqcup}_{0}$. In addition, we take Weissenberg number, $\mathrm{We}=4$, where now the characteristic velocity, $\mathrm{U}=\dot{\mathrm{\square}}_{0} \mathrm{~L}_{0}$, and for cross-reference $\mathrm{De}=\square_{1} \dot{\square}_{0}$ and $\square_{1}=2.54$ see [22].

The motivation in this part of the study is to advance this hybrid FE/FV methodology and apply the same to complex flow settings. This problem presents a number of challenging aspects including those arising in highly-deformed, moving-boundary, free-surface transient viscoelastic flows. We establish a favoured fractional-staged solution approach for this flow regime, considering various stress finite volume combinations, namely, inconsistent and consistent FV schemes, pure and hybrid FD-forms (Eq.(17-19)), and with $m d c$-inclusion (Eq.20).

\section{Free-surface computation}

The boundary conditions and geometric domain considered within the modeling are illustrated in Fig.8. In addition, initial conditions are taken as quiescent, with exception of on the moving plate, where the initial velocity is taken as $\mathrm{V}_{0}=\left(\mathrm{L}_{0} \dot{\square}_{0}\right)$. Dirichlet type boundary conditions are imposed on the known parts of the boundary. Continuity of normal and tangential velocities is applied at a fluid-solid interface and continuity of stress at a free-surface [26]. On the free boundary $\square_{\mathrm{fs}}$, considering an appropriate amount of surface-tension, we impose the dynamic and kinematic boundary conditions outlined below:

The dynamic boundary condition is given viz. 


$$
\square \cdot n=\square\left(p_{e x t}+\square\left(R_{Y}^{\square}+R_{2}^{\square 1}\right)\right) \cdot n .
$$

Here, $\square$ is the Newtonian Cauchy-stress tensor, defined as:

$$
\square_{\mathrm{ij}}=\square\left(\mathrm{p} \square_{\mathrm{ij}}+2 \square_{0} \mathrm{~d}_{\mathrm{ij}}\right)
$$

where, $\square, \mathbf{n}, \mathbf{d}$ and $p_{\text {ext }}$ are the surface tension coefficient, outward unit normal vector to the boundary

$\checkmark$, rate-of-deformation tensor and atmospheric pressure, respectively. $\mathrm{R}_{1}$ and $\mathrm{R}_{2}$ are the principal radii of curvature of the interface, $[23,27]$ defined functionally through free-surface representation $h(z, t)$,

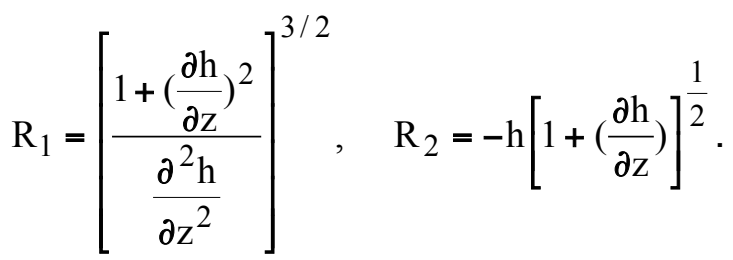

The kinematic boundary condition is taken as follows. In order to find the eventual position of the free-surface, we use

$$
\frac{\partial \mathrm{h}}{\partial \mathrm{t}}=\mathrm{u}_{\mathrm{r}} \square \mathrm{u}_{\mathrm{z}} \frac{\partial \mathrm{h}}{\partial \mathrm{z}}, \quad \square \mathrm{t}
$$

In order to retain, smoothness and consistency in surface profiles a Crank-Nicolson treatment (with $\square=0.5$ ) is introduced in discretising the second-term of r.h.s. of Eq. (31), implemented as

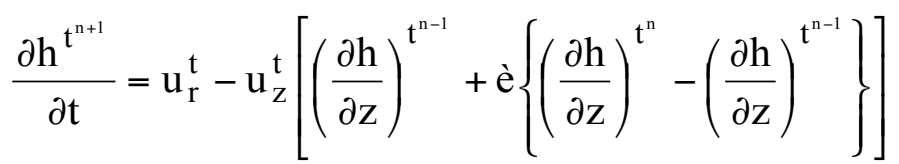

where 0 पिए1. With $\square=1$, equation is recovered, under as explicit, one-backstep interpretation between $t^{\mathrm{n}+1}$ and $\mathrm{t}^{\mathrm{n}}$. For more detail the reader is referred to [24]. Since the flow is incompressible, the free-surface location must satisfy volume conservation.

The kinematic iteration is a natural, separate solution stage for transient free-surface problems. From some initial flow field and domain, the free-surface and moving-boundary is advanced, according to the flow on such boundaries. An updated flow field is calculated and the boundaries advance oncemore, consecutively. At each step the mesh is adjusted so as to comply with kinematic conditions, (32), that match the motion of the moving boundaries to the velocity field. The kinematic iteration can be applied to solve a fully-transient problem, or simply to solve a steady state problem. Due to the underpinning boundary conditions and the presence of the free surface, the mesh experiences large deformation both radially (near the interface of the free-surface and the top-plate) and axially. Uniform remeshing is performed radially. For axial remeshing, where the mesh undergoes large stretching, a modified logarithmic (log-sec) remeshing algorithm has been employed, see reference [25] for details. 


\subsection{Results and discussion: filament-stretching}

\section{Pure FD- schemes}

To avoid ambiguity, we begin by discarding $m d c$-contributions, thus taking $\square_{\mathrm{mdc}}=0$. This implies only upwinding constructs are employed (consistently) upon flux $\left(\mathrm{R}_{\mathrm{T}}\right)$ and source $\left(\mathrm{Q}_{\mathrm{T}}\right)$ terms, as befits a highly extension-dominated problem. We focus our attention almost entirely upon the distribution of total stress (Tzz) and extra-stress $\left(\square_{z z}\right)$ along the centreline $(\mathrm{Z}=\mathrm{L}(\mathrm{t}) / 2$, see Fig. 8). For this problem, total stress is the quantity of physical interest, whilst extra stress is the primary variable computed within the FV-scheme. Here, two cell-vertex fluctuation distribution schemes of interest, LDB (linear and linearity preserving, second-order for steady problems) and Lax-Wendroff (linear, central-scheme with dissipation term, second order) are considered. Such FD-schemes are applied consistently to both flux and source terms as in Eq. (17). Along the centreline at $Z=L(t) / 2$, and for different finite volume implementations, Fig. 11 contrasts the variation of total stress $\left(T_{z z}\right)$, whilst Fig. 12 conveys the same in extra-stress $\left(\square_{z z}\right)$.

With filament stretching, when the plates move apart, free-surface deformation increases, and $\mathrm{D}_{\mathrm{zz}}$ at the mid-point of the free-surface rises sharply towards the free-surface. Hence, the total stress $\left(\mathrm{T}_{\mathrm{zz}}\right)$, elevates in a similar fashion, along the horizontal central axis and a boundary layer develops, as reported in [22]. Due to the presence of the boundary layer, the value of stress drops slightly at the interface node $\mathrm{n}_{1}$ (see Fig.8 and Fig.9) on the free-surface, when compared to its immediate neighbouring vicinity. In Fig.11, the resulting trend illustrated via the Lax-scheme agrees well with that reported by Yao and McKinley [22]. In addition, a comparison of $\mathrm{D}_{\mathrm{ZZ}}$ computed with various FV schemes is depicted in Fig. 14, contrasted against suitably scaled results from the literature [22]. For this problem, the aspect ratio of the filament is chosen as $1 / 3$ and We $=0.5$. It is apparent that the pure FD Lax-scheme outperforms the LDB and CT3-schemes. In Fig.11, the LDB-scheme almost replicates that of Lax, albeit with a slight overshoot at the left-hand end of the plot where $\mathrm{r}=0$, and undershoot at the mid-point of the free-surface (node $\mathrm{n}_{1}$ ), at the right-hand end of the plot. With the LDB-scheme, the value of extra-stress at node $n_{1}$ vanishes (see Fig. 12). The reason for the relatively poor performance of the LDB-scheme can be attributed to the prevailing direction of the radial velocity profile, $U_{r}$. In our previous study on viscous flows [24], we have shown that the flow-field at small Hencky-strains is dominated by the radial velocity, $U_{r}$, which is directed inwards at all times. Fig. 9 displays the velocity field for the half-length model. In Fig.9a, the velocity-vectors acting upon the free-surface nodes point inwards. Therefore, due to equation (17), and with the use of a pure upwinding scheme (such as LDB), it is apparent that nodes on free-surface boundaries will receive no extra-stress contribution whatsoever. This is true for mid-plane node $\left(\mathrm{n}_{1}\right)$ at all times, where vanishing axial velocity is imposed in the half-length model problem, see Fig.9b. This state of affairs applies equally to the full-length model, see Fig.10a. The transient evolution of the extra-stress $\left(\square_{\mathrm{zz}}\right)$ at the crucial node $\mathrm{n}_{1}$, the mid-point node of the free-surface, is demonstrated in Fig. 13a. This figure charts the temporal development of stress over Hencky strain levels from $\square=0$ to $\square=0.2$. We observe that with the LDB-scheme, the value of extra-stress at node $\mathrm{n}_{1}$, does not shift away from zero. Such discrepancy can be suppressed with recourse to the Lax FD-scheme (Eq.12), which imparts appropriate contributions to node $\mathrm{n}_{1}$, as desired. The transient development of extra-stress for an interior node $\mathrm{n}_{2}$ is depicted in Fig. 13b. This node is about half-way along the free-surface mid-plane from node $\mathrm{n}_{1}$ (on axis $\mathrm{Z}=\mathrm{L}(\mathrm{t}) / 2$, see Fig.9a). For this node, the LDB-result mimics the Lax-result closely throughout, and underlines the fact that discrepancy creeps in through upwinding treatment localised to the free-surface interface. 


\section{Hybrid FD-schemes}

Following our approach for model problems, we have extended our study to include various update strategies of pure FD-form, yet with hybrid combinations. Equation (17) is segregated to allow one FD-scheme to apply to $\mathrm{R}_{T}$, with a second acting on $\mathrm{Q}_{\mathrm{T}}$; so for example, $\operatorname{PSI}\left(\mathrm{R}_{\mathrm{T}}\right) / \mathrm{LDB}\left(\mathrm{Q}_{\mathrm{T}}\right)$; $\operatorname{PSI}\left(\mathrm{R}_{\mathrm{T}}\right) / \operatorname{Lax}\left(\mathrm{Q}_{\mathrm{T}}\right) ; \operatorname{Lax}\left(\mathrm{R}_{\mathrm{T}}\right) / \operatorname{PSI}\left(\mathrm{Q}_{\mathrm{T}}\right) ; \operatorname{Lax}\left(\mathrm{R}_{\mathrm{T}}\right) / \mathrm{LDB}\left(\mathrm{Q}_{\mathrm{T}}\right)$; and $\operatorname{LDB}\left(\mathrm{R}_{\mathrm{T}}\right) / \mathrm{Lax}\left(\mathrm{Q}_{\mathrm{T}}\right)$. Incorporated within Figs. 11-13, we are able to contrast results between pure and various hybrid combinations. As above, the transient development of extra-stress $\left(\square_{z z}\right)$ at the free-surface mid-plane node $\left(\begin{array}{ll}n & 1\end{array}\right)$ with various schemes is illustrated in Fig.13a. A similar plot is provided in Fig.13b at internal node $\left(\mathrm{n}_{2}\right)$ along the same axis where $\mathrm{Z}=\mathrm{L}(\mathrm{t}) / 2$. It is of interest to note that apart from minor oscillations generated by the $\operatorname{Lax}\left(\mathrm{R}_{\mathrm{T}}\right) / \mathrm{PSI}\left(\mathrm{Q}_{\mathrm{T}}\right)$ combination, all fluctuation distribution schemes behave similarly in the interior region of this central plane line, both in space and time (see Fig. 12a and Fig. 13b). Discrepancies arise close to the regions at the boundaries (see Fig. 12a and Fig. 13a). When flux terms are computed via the PSI-scheme and source terms with a Lax-stencil, the solution mimics the consistent pure-FD Lax-scheme, as illustrated in Fig. 11a and Fig. 12a. In a similar manner, PSI on flux terms and LDB on source terms, replicates the results from the pure-FD LDB-scheme. The key element here appears to be the selection of scheme for the source term $\left(\mathrm{Q}_{\mathrm{T}}\right)$. When the PSI-scheme is invoked for source terms, it introduces discretisation error and oscillations result. This is due to the positive character of the PSI-scheme, which may induce fresh extrema in the solution (see above). From the transient development of extra-stress $\left(\square_{z z}\right)$ at node $n_{1}$ in Fig. 13a, it is conspicuous that in order to have some stress contribution to the boundary node $\mathrm{n}_{1}$, the scheme of choice on $\mathrm{Q}_{\mathrm{T}}$ is that of Lax.

At the same time, the scheme applied on flux is also important. When one compares $\operatorname{Lax}\left(\mathrm{R}_{\mathrm{T}}\right) / \operatorname{Lax}\left(\mathrm{Q}_{\mathrm{T}}\right)$ versus $\mathrm{LDB}\left(\mathrm{R}_{\mathrm{T}}\right) / \mathrm{Lax}\left(\mathrm{Q}_{\mathrm{T}}\right)$ in Fig.13a, the $\mathrm{LDB}$-version on $\mathrm{R}_{\mathrm{T}}$, generates negatives values for extra-stress at the outset and through the Lax-form on $\mathrm{Q}_{\mathrm{T}}$, the node subsequently gathers contribution in time (solution undershoot results). Note that, with $\mathrm{LDB}$ alone on $\mathrm{R}_{\mathrm{T}}$ and $\mathrm{Q}_{\mathrm{T}}$, no contribution to the node $\mathrm{n}_{1}$ is delivered. It would appear that in the region near the free-surface, both flux and source terms are of comparable strength, and the LDB-scheme when used on either flux or source, degrades the solution. In contrast, the PSI on $\mathrm{R}_{\mathrm{T}}$ with $\mathrm{Lax}$ on $\mathrm{Q}_{\mathrm{T}}$, replicates the solution exactly of $\operatorname{Lax}\left(\mathrm{R}_{\mathrm{T}}\right) / \operatorname{Lax}\left(\mathrm{Q}_{\mathrm{T}}\right)$. So positivity via PSI does not detract from $\mathrm{R}_{\mathrm{T}}$-treatment, once $\mathrm{Lax}$ is imposed on $\mathrm{Q}_{\mathrm{T}}$, and yields high precision results in contrast to LDB. This proves the point made earlier on model problems, the essential contribution of positivity upon flux terms, here illustrated through time accuracy (as true for Lax- $\mathrm{R}_{\mathrm{T}}$ implementation, also). Furthermore, note that once Lax $\left(\mathrm{Q}_{\mathrm{T}}\right)$ is replaced with $\operatorname{LDB}\left(\mathrm{Q}_{\mathrm{T}}\right)$, the $\operatorname{PSI}\left(\mathrm{R}_{\mathrm{T}}\right)$ influence is dominated by the source effect, to the extent that there is now no contribution to extra-stress once again. Hence, pure upwinding performs poorly, on $\mathrm{Q}_{\mathrm{T}}$-terms. Alternatively, the Lax-scheme performs better with $\mathrm{Q}_{\mathrm{T}}$-terms as it is designed to provide second-order accuracy in time for purely convective flows. One notes the structure of the Lax-scheme from Eq.(12) and the perturbation form about the uniform 1/3-weighting (hence, not purely upwinding by definition).

\section{Schemes with mdc-inclusion}

Elsewhere for complex flows, $m d c$-contributions have been found to ensure numerical stability and accuracy for both steady and transient problems $[2,12,13]$. With $m d c$ inclusion, the scheme employed here is $\mathrm{CT}_{3}$, the more consistent form with $\square_{\mathrm{mdc}}=1 \square_{\mathrm{t}}$ [12]. In the present transient problem, from Fig. $11 \mathrm{~b}$ and Fig. 12b, it is apparent that the inclusion of $m d c$ degrades the solution. The inclusion of $m d c$-contributions diffuses the solution (see Fig. 12b). The discrepancy is greater at the free-surface and the central region, where the velocity gradient $\mathrm{D}_{\mathrm{zz}}$, rises sharply from a constant value to a peak, as demonstrated in Fig. 14. In the regions where such sharp change in gradient occurs, the strength of the source terms can be high and the flow can be heavily convective in nature. The adjustment in direction of flow is apparent in the velocity vectors in Fig. 9. Hence, 
fluctuation distribution alone is superior to only $m d c$-inclusion. The inclusion of $m d c$ introduces significant error in the transient stress evolution at the free-surface node $\mathrm{n}_{1}$, and the internal node $\mathrm{n}_{2}$, as demonstrated in Fig. 13a and Fig. 13b. The value of extra-stress at the free-surface node $\mathrm{n}_{1}$, is always negative with $m d c$-inclusion. Fig $11 \mathrm{~b}$ contrasts Lax and LDB implementations, both with $m d c$-inclusion $\left(\mathrm{CT}_{3}\right)$. Here, the linearity preserving property of LDB preserves greater smoothness in the solution. Nevertheless, $m d c$-inclusion is clearly responsible for greater injection of error in the solution, irrespective of Lax or LDB choice. In addition, we have considered variants of the standard inconsistent $\left(\square \mathrm{R}_{\mathrm{T}}+\mathrm{Q}_{\mathrm{mdc}}\right)$ scheme, and $\left(\square \mathrm{R}_{\mathrm{T}}+\square_{\mathrm{T}} \mathrm{Q}_{\mathrm{T}}+\square_{\mathrm{mdc}} \mathrm{Q}_{\mathrm{mdc}}\right)$-scheme. Here, $\mathrm{R}_{\mathrm{T}}$ is of Lax-form.

The results are displayed in Fig. $11 \mathrm{~b}$ and Fig. 12b, from which it is conspicuous that for this problem, any form of $m d c$-inclusion, degrades the solution, irrespective of whether this is imparted to source terms alone or with flux terms, consistently or inconsistently. Curiously, the standard implementation (S1) does provide the most damping to the interior solution profile in Fig. 11b.

\section{Conclusion}

For model problems, consistent formulation of the flux and source terms has been found to improve the accuracy to almost a third-order. The precise choice of a FD-scheme for the flux and source terms is the key issue, in attaining such higher orders of accuracy. An inappropriate choice would detract from this position. In shifting from structured to unstructured meshes, one order of accuracy is lost. Through the study of various FV-scheme choices upon a well-characterised model problem, we have been able to pinpoint some of the key issues that must be addressed in accurately discretising viscoelastic flow equations. The principle issue has been the consistent incorporation of non-trivial source terms within a standard FV cell-vertex formulation. Overall, we conclude for model flows, that consistency of approach is vital to attain the higher levels of accuracy, be this on structured or unstructured meshes. Under optimal selection, this may yield a third-order of accuracy on structure meshes and second-order on unstructured alternatives. In contrast, for standard approaches, such as inconsistent scheme S1 (commonly quoted in the literature), we have illustrated how a significant loss of accuracy may be incurred. The consequence is restriction to first-order accuracy. This may give rise to other, knock-on effects in the accurate resolution of more complex, highly-elastic flows.

In the study of complex problems under filament-stretching flows, we have demonstrated improved levels of accuracy achieved with a Lax-scheme. This is so, in contrast to a counterpart LDB-implementation, reported at small Hencky-strain levels. Here, we have been able to isolate the shortcomings of these upwinding schemes, whilst dealing with such transient free-surface problems. We observe that the velocity-field in these filament-stretching flows with liquid-bridges imposes certain restrictions on the upwinding scheme approximations for these to perform well in the presence of free-surfaces. An optimal choice has been proposed, having contrasted results for pure fluctuation distribution schemes, various hybrid combinations, and with $m d c$-inclusion. We summarise our main conclusions. First, $m d c$-inclusion is not recommended for such transient extension-dominated problems with contact-line/interfaces, as it degrades accuracy. High accuracy is achieved via the use of the second-order Lax-scheme. The optimal choice for such transient contact-line/interface problems is Lax on $\mathrm{Q}_{\mathrm{T}}$; on $\mathrm{R}_{\mathrm{T}}$, Lax or PSI. Application of positive schemes on $\mathrm{Q}_{\mathrm{T}}$ degrades the solution for both model and complex problems. We have been able to achieve a high degree of precision on velocity gradients with pointwise estimation, even accommodating contact-line interfaces. The results obtained with the optimum scheme, agree well with counterpart finite element solutions available in the open literature. 


\section{References}

1 H. Matallah, P. Townsend and M. F. Webster, Recovery and stress-splitting schemes for viscoelastic flows, J. Non-Newt. Fluid Mech., 75: 139-166 (1998).

2 P. Wapperom and M. F. Webster, Simulation for viscoelastic flow by a finite volumelelement method, Comp. Meth. Appl. Mech. Eng., 180: 281-304 (1999).

3 M. S. Darwish and J. R. Whiteman, Numerical modelling of viscoelastic liquids using finite-volume method, J. Non-Newt. Fluid Mech., 45: 311-337, (1992).

4 M. E. Hubbard and P.L Roe, Multidimensional upwind fluctuation distribution schemes for scalar time dependent problems, Int. J. Num. Meth. Fluids, 33:711-736 (2000)

5 K. W. Morton, P. I. Crumpton and J. A. Mackenzie, Cell-vertex methods for inviscid and viscous flows, Comp. Fluids, 22: 91-102 (1993).

6 P. I. Crumpton, J. A. Mackenzie and K. W. Morton, Cell-vertex algorithms for the compressible Navier-Stokes equations, J. Comp. Phys., 109: 1-15 (1993).

7 K.W. Morton, M.A. Rudgyard, and G. J. Shaw, Upwind iteration methods for the cell vertex scheme in one dimension, J. Comp. Phys., 114: 209-226 (1994).

8 M. Berzins and J. M. Ware, Positive cell-centered finite volume discretization methods for hyperbolic equations on irregular meshes, Appl. Num. Meths., 16: 417-438 (1995).

9 R. Struijs, H. Deconink, P. L. Roa, Fluctuation splitting for the 2D Euler equations, Technical Report Series, von Karman Institute of Fluid Dynamics (1991).

10 T. Sato and S. M. Richardson, Explicit numerical simulation of time-dependent viscoelastic flow problems by finite element/finite volume method, J. Non-Newt. Fluid Mech., 51: 249-275 (1994).

11 A. N. Brooks and J.R. Hughes, Streamline upwind/Petrov-Galerkin formulations for convection dominated flows with particular emphasis on the incompressible Navier-Stokes equations, Comp. Meth. Appl. Mech. Eng., 32: 199-259 (1982).

12 M. Aboubacar and M. F. Webster, Development of an optimal hybrid finite volume/finite element method for viscoelastic flows, Int. J. Num. Meth. Fluids, 41, 1147-1172 (2003).

13 M. F. Webster, H. R. Tamaddon-Jahromi and M. Aboubacar, Transient viscoelastic flows in planar contractions, J. Non-Newt. Fluid Mech. (in press) (2003).

14 J. G. Oldroyd, On the formulation of rheological equations of state, Proc. Roy. Soc., A200: 523541 (1950).

15 C. Hirsh, Numerical simulations of internal and external flows, vol. 2, Wiley (1990).

16 S. Gunter, P. Townsend and M.F. Webster, The simulation of some model viscoelastic flows, Int. J. Num. Meth. Fluids, 23:691-710 (1996).

17 S. H. Spiegelberg, D. C. Ables, G. H. McKinley, The role of end-effects on measurements of extensional viscosity in filament stretching rheometers, J. Non-Newt. Fluid Mech., 64:229-267 (1996).

18 V. Tirtaatmadja and T. Sridhar, A filament stretching device for measurement of extensional viscosity, J. Rheol., 37:1081-1102 (1993).

M. Yao, G. H. McKinley and B. Debbaut, Extensional deformation, stress relaxation and necking failure of viscoelastic filaments, J. Non-Newt. Fluid Mech., 79:469-501 (1998).

20 R. Sizaire and V. Legat, Finite Element simulation of a filament stretching extensional rheometer, J. Non-Newt. Fluid Mech., 71:89-107 (1997).

21 H. K. Rasmussen and O. Hassager, Three-dimensional simulations of viscoelastic instability in polymeric filament, J. Non-Newt. Fluid Mech., 82:189-202 (1999).

M. Yao and G. H. McKinley, Numerical simulation of extensional deformations of viscoelastic liquid bridges in filament stretching devices, J. Non-Newt. Fluid Mech., 74:47-88 (1998).

23 P. Tanguay, M. Fortin and L. Choplan, Finite element simulation of dip coating, I: Newtonian Fluids, Int. J. Num. Meth. Fluids, 4: 441-457 (1984).

24 M. S. Chandio, H. Matallah and M. F. Webster, Numerical Simulation of Viscous FilamentStretching flows, Int. J. Num. Meth. Heat Fluid Flow, (in press) (2003). 
R. Kröger, S. Berg, A. Delgado and H. J. Rath, A stretching behaviour of large polymeric and Newtonian liquid bridges in plateau simulation, J. Non-Newt. Fluid. Mech., 45: 385-400 (1992).

26 E. Fourcade, F. Bertrand, O. Reglat and P. A. Tanguy, Finite element analysis of fluid-solid interaction in the metering nip of a metering size press, Comput. Meth. Appl. Mech. Eng., 174:235245 (1999).

27 R. Keunings. An algorithm for the simulation of transient viscoelastic flows with free surfaces, J. Comp. Phys., 20:209-226 (1986).

28 J. Donea, A Taylor-Galerkin method for convective transport problems, Int. J. Num. Meth. Eng., 20: 101-119 (1984). 


\section{List of Tables}

1. $\left\|\square \square_{\mathrm{rr}}\right\|$ data, structured meshes, consistent versus inconsistent schemes

2. $\left\|\mathrm{D}_{\mathrm{rr}}\right\|$ data: unstructured meshes, consistent versus inconsistent schemes

3. $\left\|\square_{\mathrm{rr}}\right\|$ data: unstructured meshes, consistent, LDB versus PSI.

4. $\left\|\square \square_{\mathrm{rr}}\right\|$ data: unstructured meshes, consistent versus inconsistent, LDB, PSI.

\section{List of Figures}

1. (a) FE with $4 \mathrm{FV}$ sub-cells, (b) mdc area for node $l$.

2. Schematic diagram of 2-D sink flow.

3. Structured meshes: (a) $5 \times 5$, (b) $10 \times 5$, (c) $20 \times 5$ and (d) $40 \times 5$.

4. Unstructured meshes: (a) $5 \times 5$, (b) $10 \times 10$, (c) $20 \times 20$ and (d) $40 \times 40$.

5. $\quad \mathrm{C}_{\mathrm{r}} \mathrm{h}$-convergence plots for Oldroyd-B; structured meshes: (a) consistent treatment, (b) inconsistent treatment, flux and source, unstructured meshes: (c) consistent treatment, (d) inconsistent treatment, flux and source.

6. $\square_{\mathrm{r}} \mathrm{h}$-convergence plots for Oldroyd-B: unstructured meshes;
(a) $\square_{\mathrm{l}(\mathrm{psi})}^{\mathrm{T}}\left(\mathrm{R}_{\mathrm{T}}+\mathrm{Q}_{\mathrm{T}}\right)$
(b) $\square_{1(\mathrm{psi})}^{\mathrm{T}} \mathrm{R}_{\mathrm{T}}+\square_{1(\mathrm{ldb})}^{\mathrm{T}} \mathrm{Q}_{\mathrm{T}}$, (c)
(c) $\square_{1(1 \mathrm{db})}^{\mathrm{T}} \mathrm{R}_{\mathrm{T}}+\square_{1(\mathrm{psi})}^{\mathrm{T}} \mathrm{Q}_{\mathrm{T}}$

7. $\quad \mathrm{T}_{\mathrm{r}}$ line plots: unstructured meshes; sampling inlet boundary-node ( $\mathrm{r}=1.8, \mathrm{z}=0.9$ ), (a) $5 \times 5$ mesh, (b) $40 \times 40$ mesh; sampling central-node ( $\mathrm{r}=1.5, \mathrm{z}=0.5)$; (c) $5 \times 5$ mesh, (d) $40 \times 40$ mesh.

8. $\quad$ Domain of filament, full length model with boundary conditions.

9. Velocity field: half-length model, (a) $\square=0.32$, (b) $\square=1.6$, normal and zoomed views, Newtonian liquid-bridge.

10. Velocity field: full-length model, (a) $\square=0.32$, (b) $\square=1.6$, normal and zoomed views, Newtonian liquidbridge.

11. Total stress $\mathrm{T}_{\mathrm{zZ}}$, along centre-line at $\mathrm{Z}=\mathrm{L}(\mathrm{t}) / 2, W e=4$, initial aspect ratio $\square_{0}=\frac{1}{3}$ : (a) different $\mathrm{FD}$ schemes; (b) mdc-inclusion.

12. Extra stress $\square_{\mathrm{ZZ}}$, along centre-line at $\mathrm{Z}=\mathrm{L}(\mathrm{t}) / 2, W e=4$, initial aspect ratio $\square_{0}=\frac{1}{3}$ : (a) pure and hybrid fluctuation distribution FV- schemes; (b) mdc-inclusion.

13. Transient development of extra stress $\square_{\mathrm{zz}},-=0$ to 0.2 , at (a) node $\mathrm{n}_{1}$, (b) node $\mathrm{n}_{2}$

14. Comparison of $\mathrm{D}_{\mathrm{zz}}$ along $\mathrm{Z}=\mathrm{L}(\mathrm{t}) / 2$, different schemes versus literature, initial aspect ratio $=1 / 3, \mathrm{We}=0.5$ 


\begin{tabular}{|c|c|c|c|}
\hline \multicolumn{2}{|c|}{ method $/$ mesh } & Consistent & Inconsistent \\
\hline \multirow[t]{4}{*}{ S1 } & $5 \times 5$ & $0.2510^{-1}$ & $0.6510^{-1}$ \\
\hline & $10 \times 5$ & $0.9910^{-2}$ & $0.3510^{-1}$ \\
\hline & $20 \times 5$ & $0.5010^{-2}$ & $0.1910^{-1}$ \\
\hline & $40 \times 5$ & $0.2510^{-2}$ & $0.9510^{-2}$ \\
\hline \multirow[t]{4}{*}{$\mathrm{S} 2$} & $5 \times 5$ & $0.7510^{-2}$ & $0.2810^{-1}$ \\
\hline & $10 \times 5$ & $0.1710^{-2}$ & $0.1710^{-1}$ \\
\hline & $20 \times 5$ & $0.4210^{-3}$ & $0.9710^{-2}$ \\
\hline & $40 \times 5$ & $0.1010^{-3}$ & $0.5210^{-2}$ \\
\hline \multirow[t]{4}{*}{$\mathrm{S} 3$} & $5 \times 5$ & $0.3210^{-1}$ & $0.6210^{-1}$ \\
\hline & $10 \times 5$ & $0.9210^{-2}$ & $0.3710^{-1}$ \\
\hline & $20 \times 5$ & $0.2610^{-2}$ & $0.2310^{-1}$ \\
\hline & $40 \times 5$ & $0.3910^{-3}$ & $0.1310^{-1}$ \\
\hline
\end{tabular}

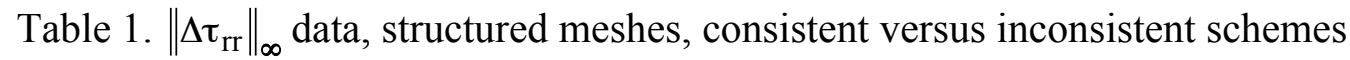

\begin{tabular}{|l|r|l|l|}
\hline \multicolumn{2}{|c|}{ method/mesh } & Consistent & Inconsistent \\
\hline S1 & $5 \times 5$ & $0.2510^{-1}$ & $0.6610^{-1}$ \\
& $10 \times 10$ & $0.7910^{-2}$ & $0.3810^{-1}$ \\
& $20 \times 20$ & $0.2510^{-2}$ & $0.2810^{-1}$ \\
& $40 \times 40$ & $0.7410^{-3}$ & $0.1510^{-1}$ \\
\hline S2 & $5 \times 5$ & $0.5310^{-2}$ & $0.3710^{-1}$ \\
& $10 \times 5$ & $0.1410^{-2}$ & $0.2510^{-1}$ \\
& $20 \times 5$ & $0.5410^{-3}$ & $0.1410^{-1}$ \\
& $40 \times 5$ & $0.2910^{-3}$ & $0.7710^{-2}$ \\
\hline S3 & $5 \times 5$ & $0.2610^{-1}$ & $0.6810^{-1}$ \\
& $10 \times 5$ & $0.6610^{-2}$ & $0.5010^{-1}$ \\
& $20 \times 5$ & $0.3010^{-2}$ & $0.6010^{-1}$ \\
& $40 \times 5$ & $0.7110^{-3}$ & 0.1409 \\
\hline
\end{tabular}

Table 2. \|GGrr $\|$ data: unstructured meshes, consistent versus inconsistent schemes 


\begin{tabular}{|l|r|l|l|}
\hline \multicolumn{2}{|c|}{ method/mesh } & Consistent(LDB & Consistent (PSI) \\
\hline S1 & $5 \times 5$ & $0.2510^{-1}$ & $0.2410^{-1}$ \\
& $10 \times 10$ & $0.7910^{-2}$ & $0.7810^{-2}$ \\
& $20 \times 20$ & $0.2510^{-2}$ & $0.2510^{-2}$ \\
& $40 \times 40$ & $0.7410^{-3}$ & $0.7410^{-3}$ \\
\hline S2 & $5 \times 5$ & $0.5310^{-2}$ & $0.5210^{-2}$ \\
& $10 \times 5$ & $0.1410^{-2}$ & $0.1410^{-2}$ \\
& $20 \times 5$ & $0.5410^{-3}$ & $0.5410^{-3}$ \\
& $40 \times 5$ & $0.2910^{-3}$ & $0.2910^{-3}$ \\
\hline S3 & $5 \times 5$ & $0.2610^{-1}$ & $0.2610^{-1}$ \\
& $10 \times 5$ & $0.6610^{-2}$ & $0.6810^{-2}$ \\
& $20 \times 5$ & $0.3010^{-2}$ & $0.3110^{-2}$ \\
& $40 \times 5$ & $0.7110^{-3}$ & $0.7210^{-3}$ \\
\hline
\end{tabular}

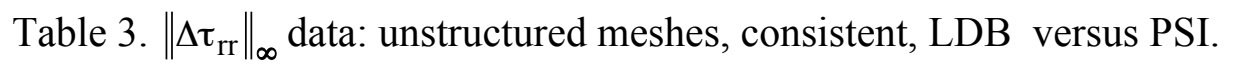

\begin{tabular}{|l|r|l|l|}
\hline \multicolumn{2}{|r|}{ method/mesh } & & \\
\hline S1 & $5 \times 5$ & $0.2510^{-1}$ & $0.2610^{-1}$ \\
& $10 \times 10$ & $0.7310^{-2}$ & 0.15 \\
& $20 \times 20$ & $0.2210^{-2}$ & 0.11 \\
& $40 \times 40$ & $0.6110^{-3}$ & 0.11 \\
\hline S2 & $5 \times 5$ & $0.5310^{-2}$ & $0.7210^{-2}$ \\
& $10 \times 5$ & $0.1110^{-2}$ & $0.2910^{+1}$ \\
& $20 \times 5$ & $0.5010^{-3}$ & 0.12 \\
& $40 \times 5$ & $0.2110^{-3}$ & 0.10 \\
\hline S3 & $5 \times 5$ & $0.2610^{-1}$ & $0.1410^{+1}$ \\
& $10 \times 5$ & $0.4410^{-2}$ & $0.1810^{+1}$ \\
& $20 \times 5$ & $0.2610^{-2}$ & $0.4110^{+1}$ \\
& $40 \times 5$ & $0.6110^{-3}$ & $0.5510^{+1}$ \\
\hline
\end{tabular}

Table 4. \|DGrr $\|$ data: unstructured meshes, consistent versus inconsistent, LDB, PSI. 


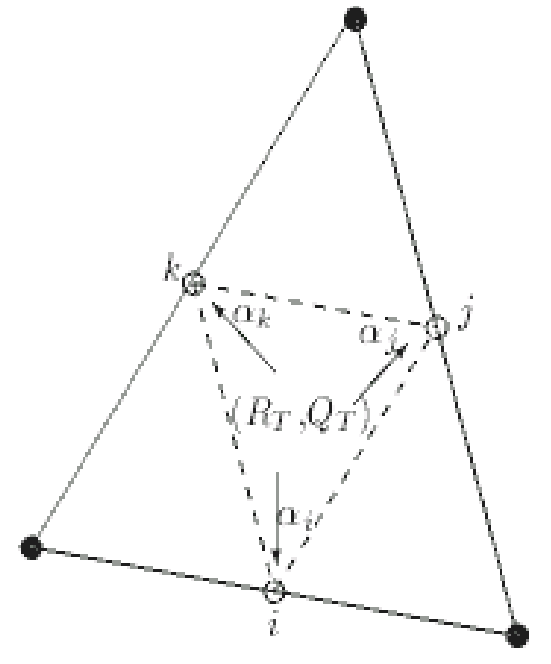

(a)

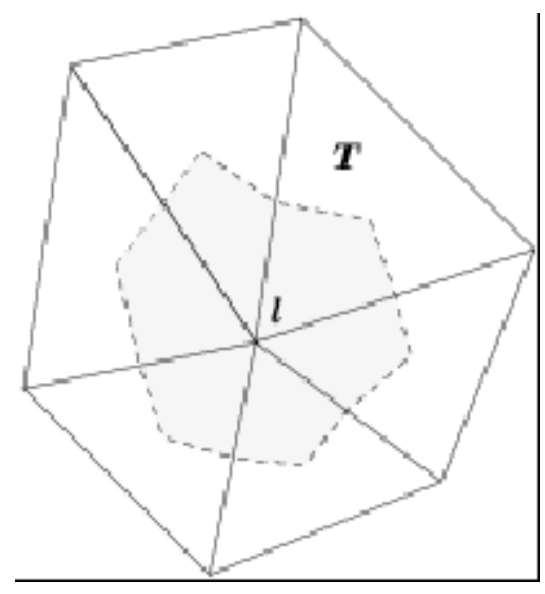

(b)

Figure 1. (a) FE with 4 FV sub-cells, (b) mdc area for node $l$.

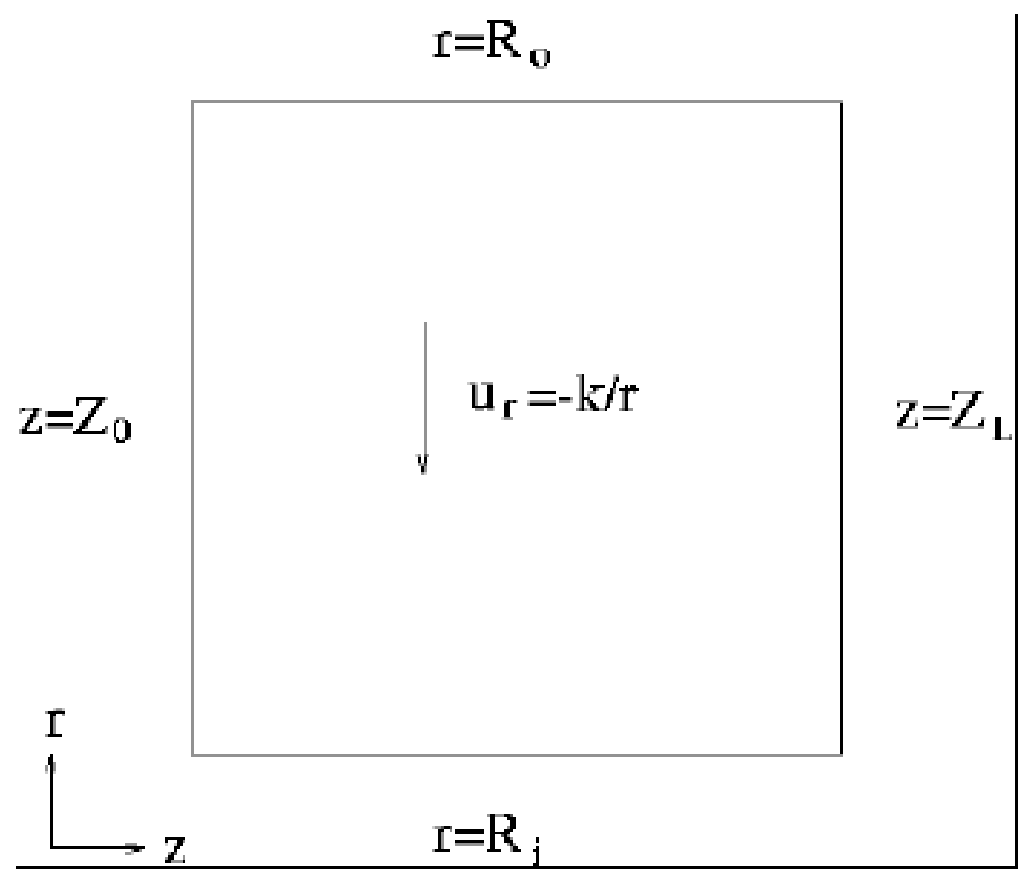

Figure 2. Schematic diagram of 2-D sink flow. 


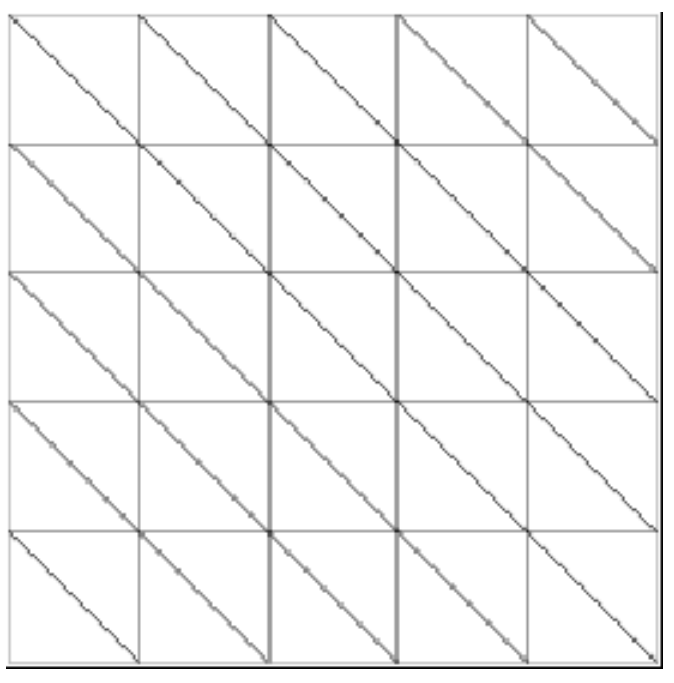

(a)

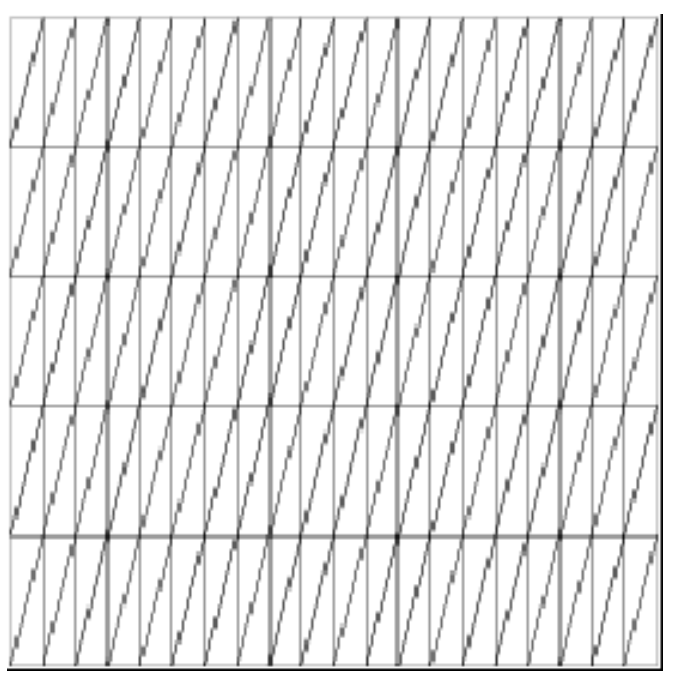

(c)

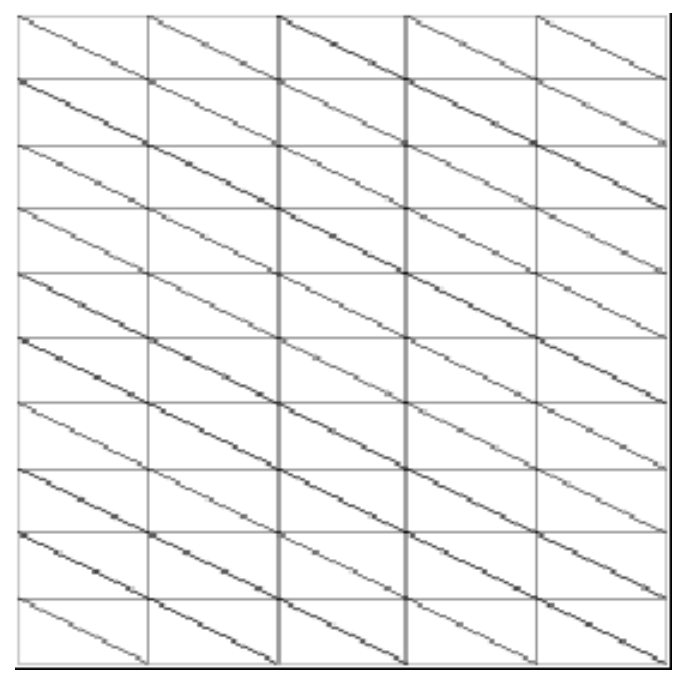

(b)

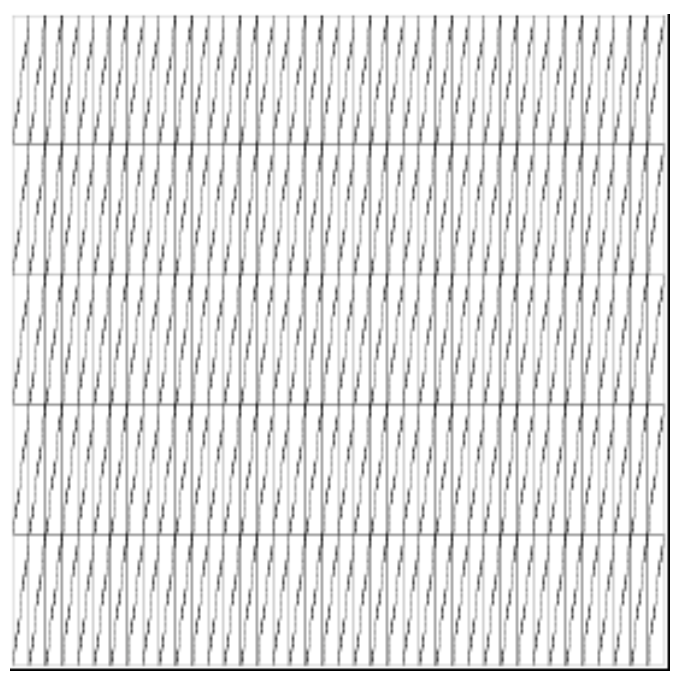

(d)

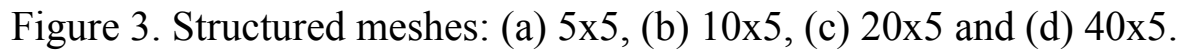




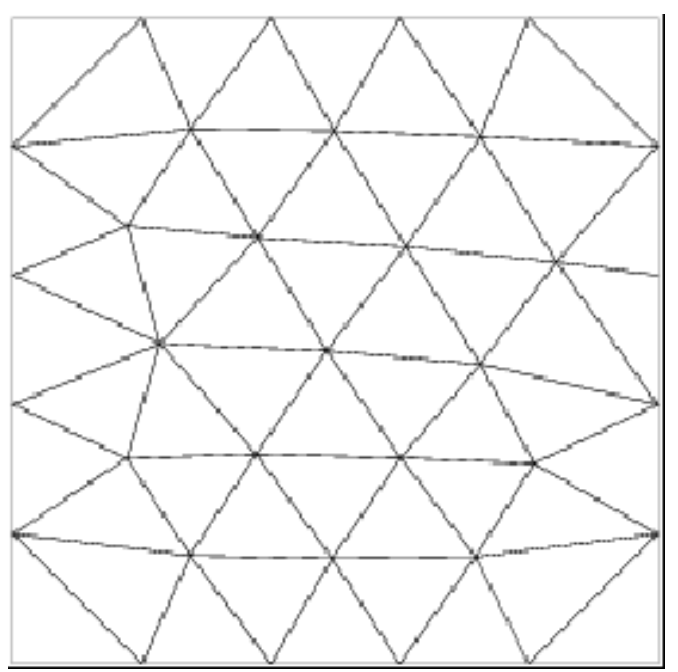

(a)

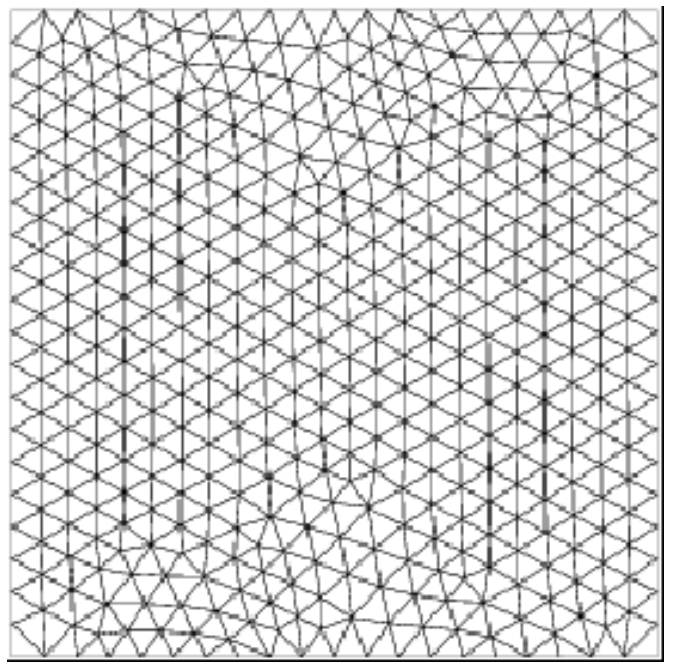

(c)

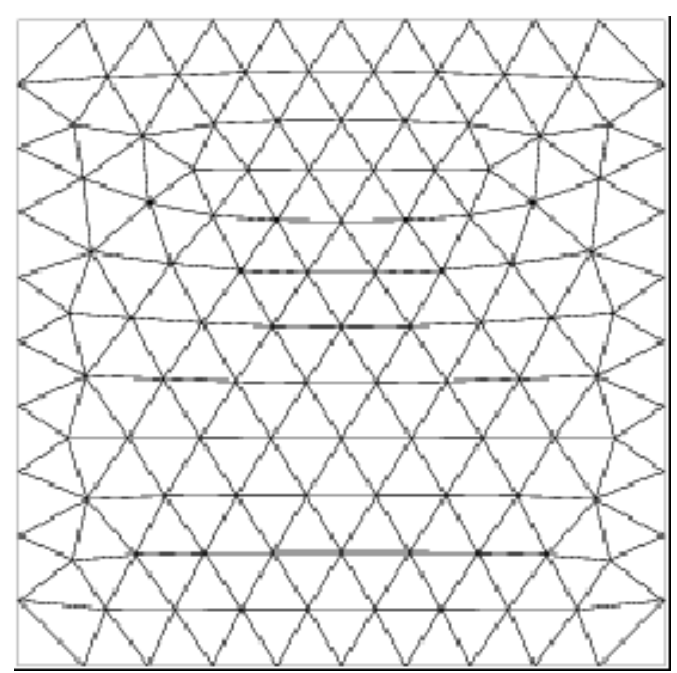

(b)

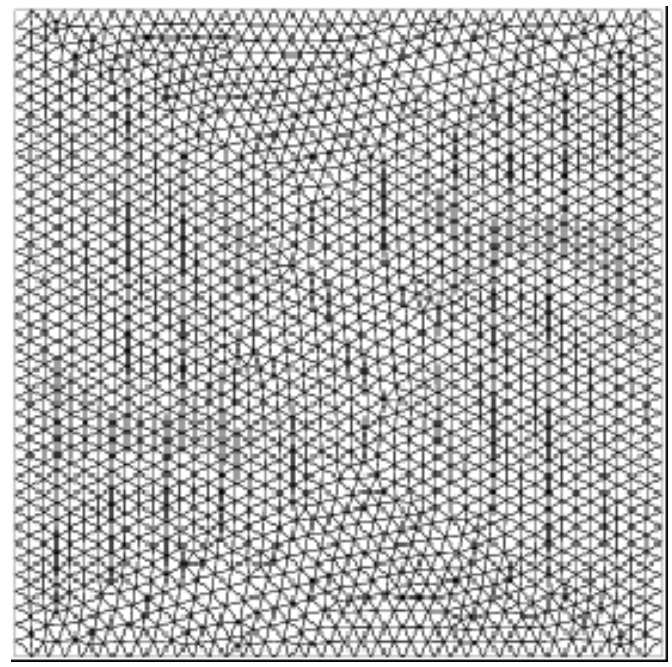

(d)

Figure 4. Unstructured meshes: (a) 5x5, (b) 10x10, (c) 20x20 and (d) 40x40. 


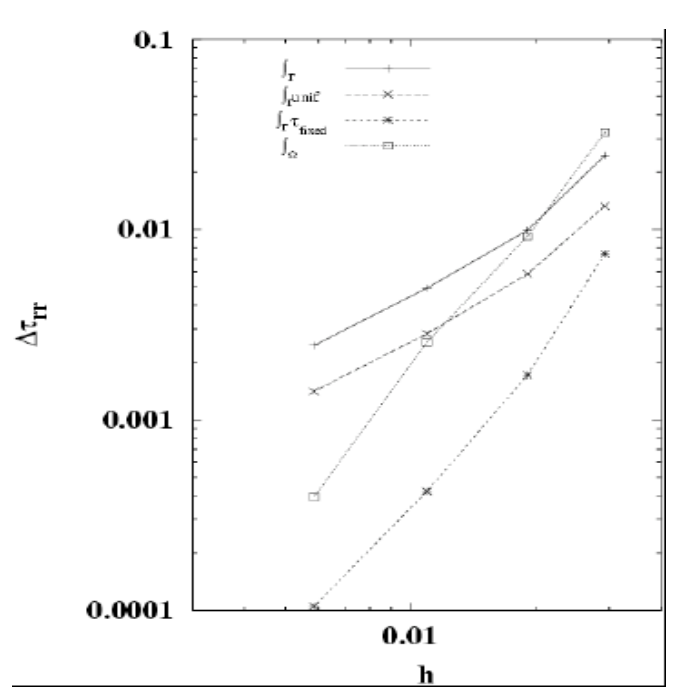

(a)

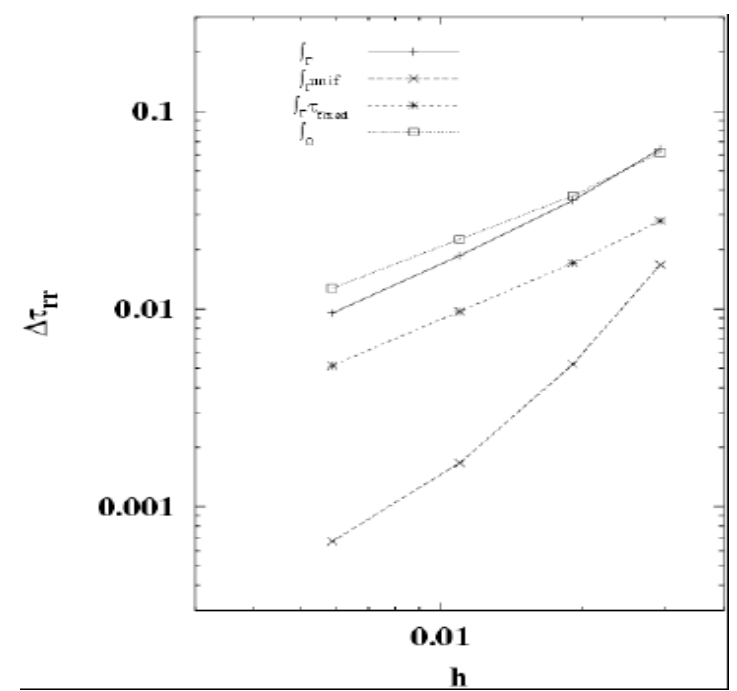

(b)

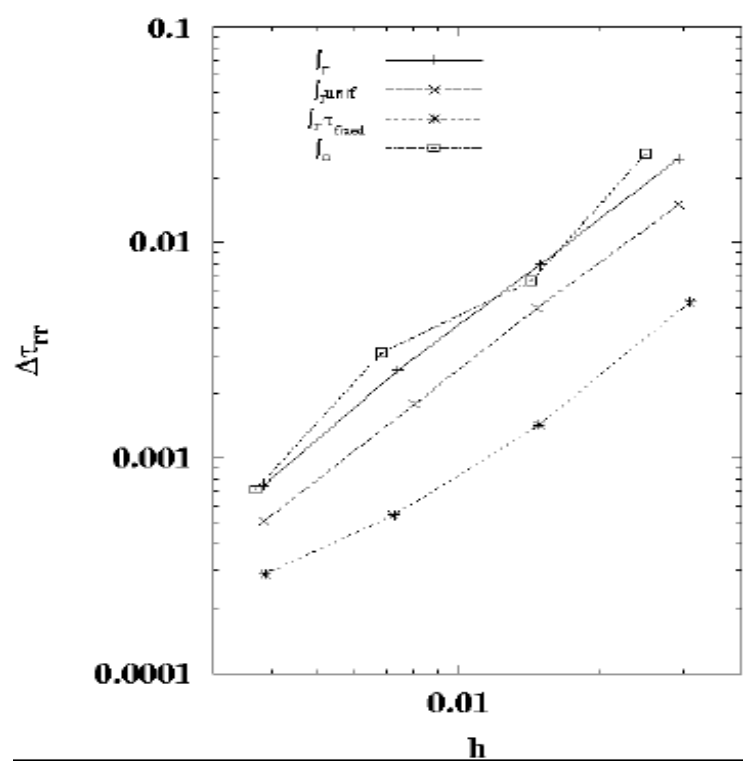

(c)

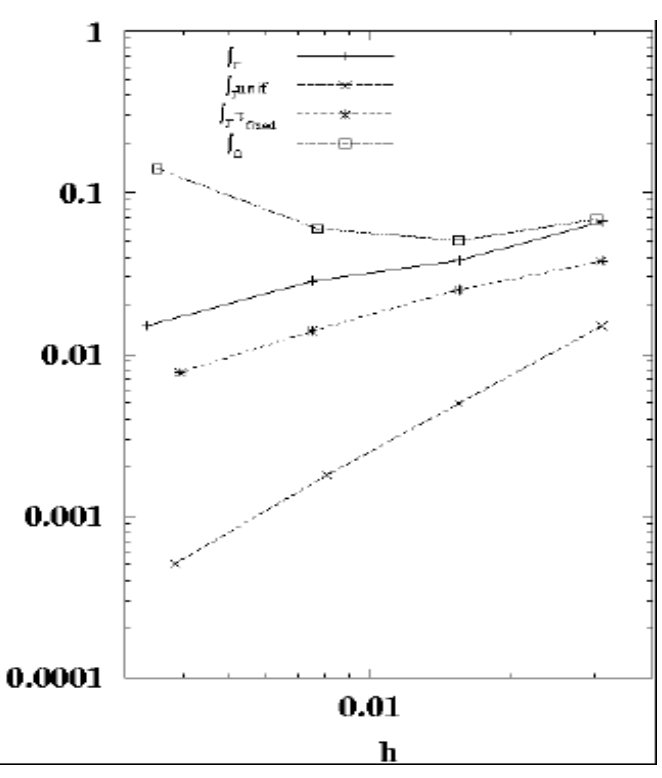

(d)

Figure 5. $\mathrm{CG}_{\mathrm{r}}$ h-convergence plots for Oldroyd-B; structured meshes: (a) consistent treatment, (b) inconsistent treatment, flux and source, unstructured meshes: (c) consistent treatment, (d) inconsistent treatment, flux and source. 


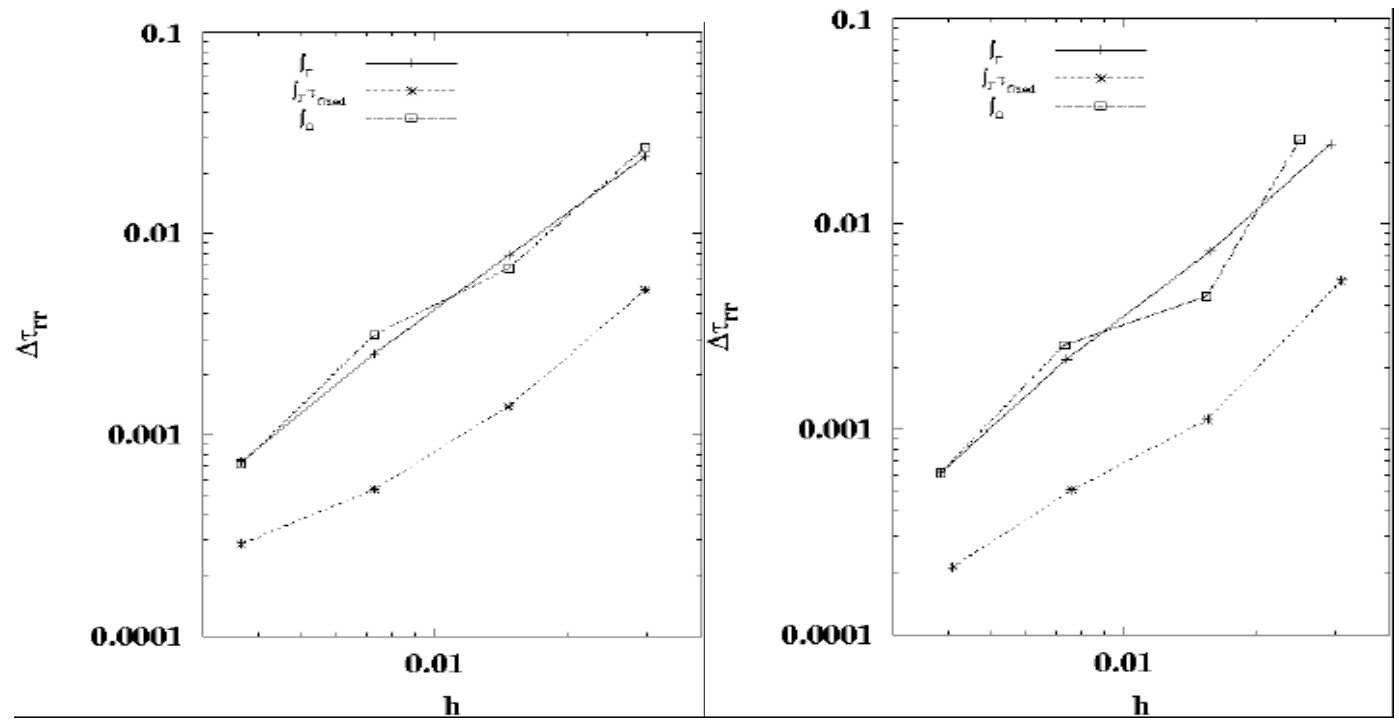

(a)

(b)

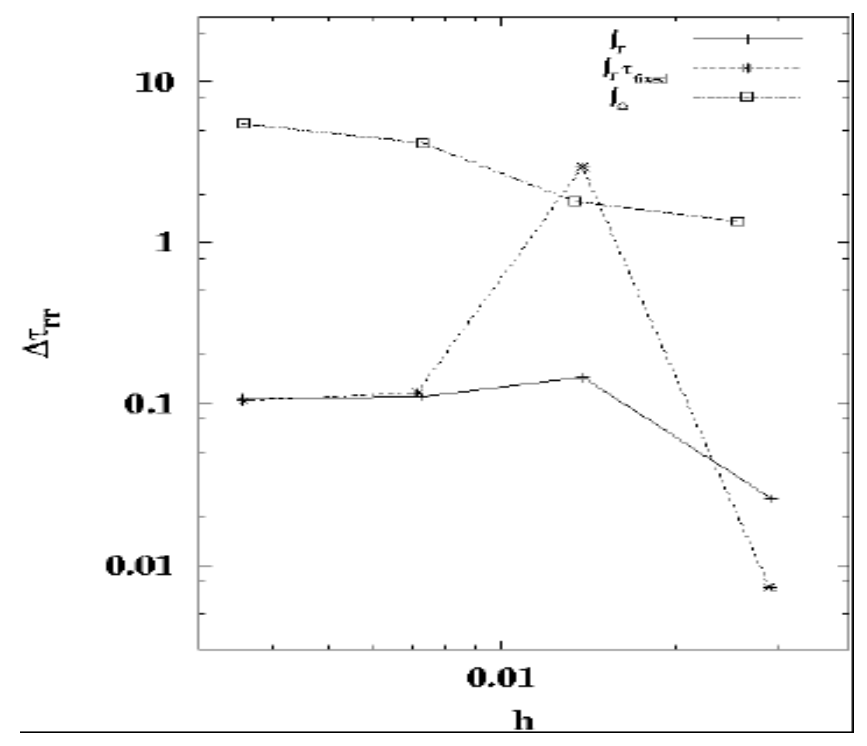

(c)

Figure 6. $D_{\mathrm{r}}$ h-convergence plots for Oldroyd-B: unstructured meshes;

(a) $\square_{1(\mathrm{psi})}^{\mathrm{T}}\left(\mathrm{R}_{\mathrm{T}}+\mathrm{Q}_{\mathrm{T}}\right)$ (b) $\square_{1(\mathrm{psi})}^{\mathrm{T}} \mathrm{R}_{\mathrm{T}}+\square_{1(1 \mathrm{db})}^{\mathrm{T}} \mathrm{Q}_{\mathrm{T}}$, (c) $\square_{1(1 \mathrm{ld})}^{\mathrm{T}} \mathrm{R}_{\mathrm{T}}+\square_{1(\mathrm{psi})}^{\mathrm{T}} \mathrm{Q}_{\mathrm{T}}$ 


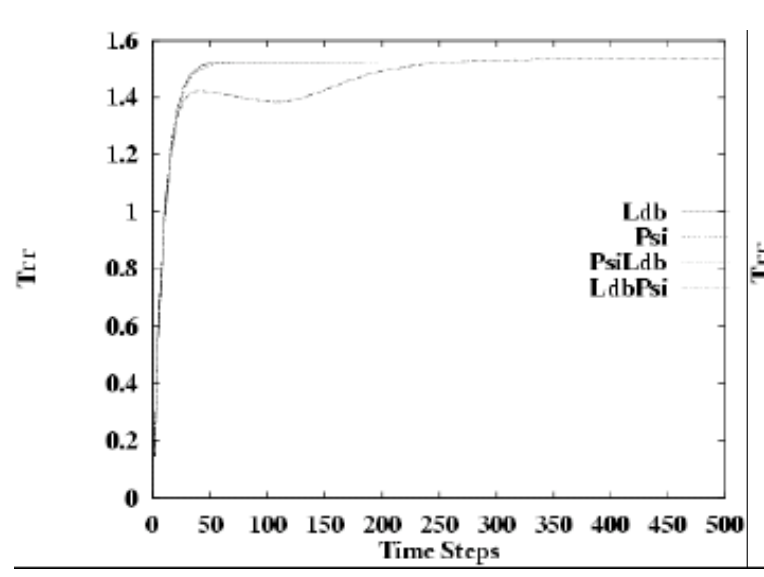

(a) $5 \times 5$

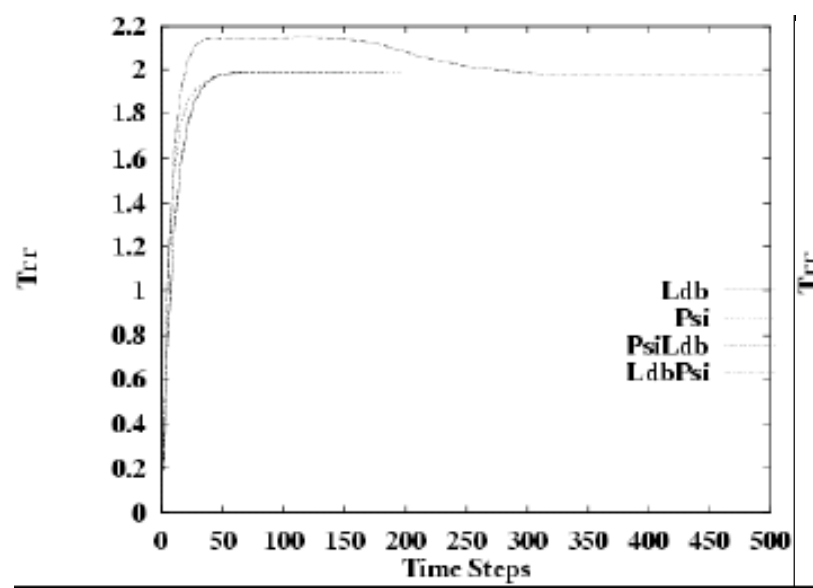

(c) $5 \times 5$

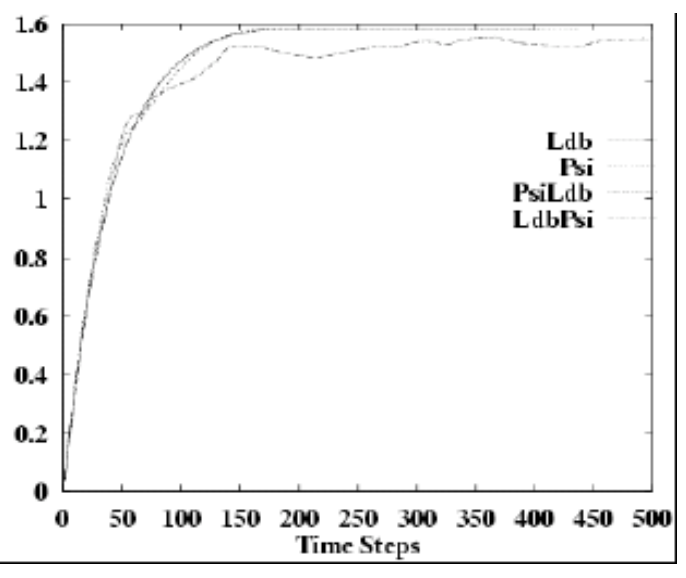

(b) $40 \times 40$

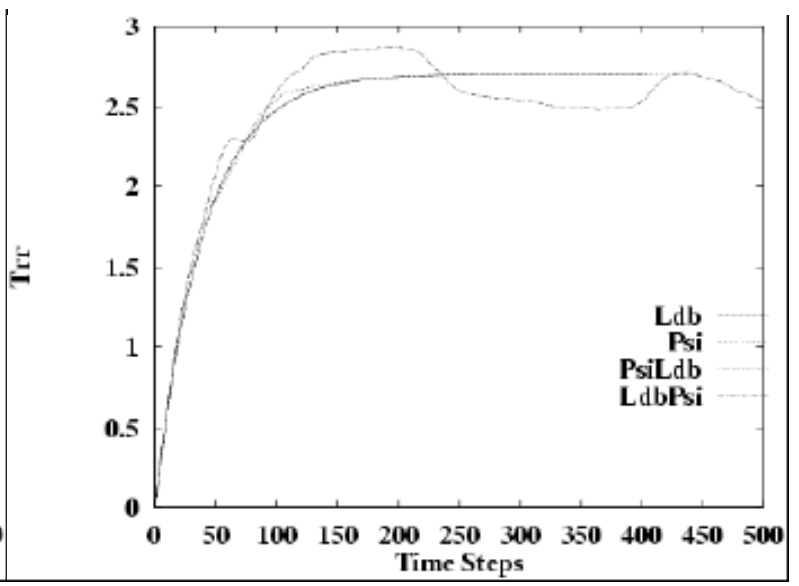

(d) $40 \times 40$

Figure 7. $\square_{r}$ line plots, unstructured meshes;

sampling inlet boundary-node $(\mathrm{r}=1.8, \mathrm{z}=0.9)$; (a) 5x5 mesh, (b) 40x40 mesh; sampling central-node ( $\mathrm{r}=1.5, \mathrm{z}=0.5)$; (c) $5 \times 5$ mesh, (d) 40x40 mesh. 


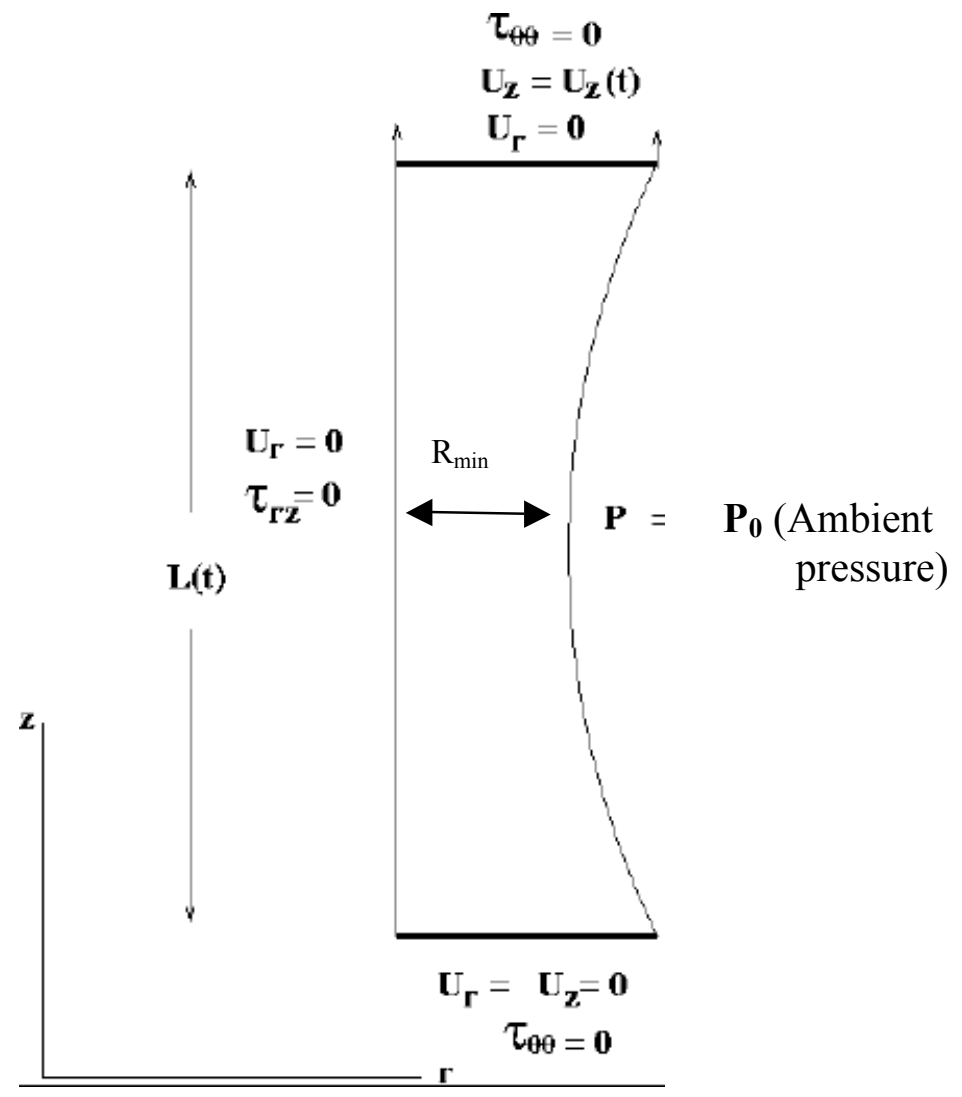

Figure 8 . Domain of filament, full length model with boundary conditions. 

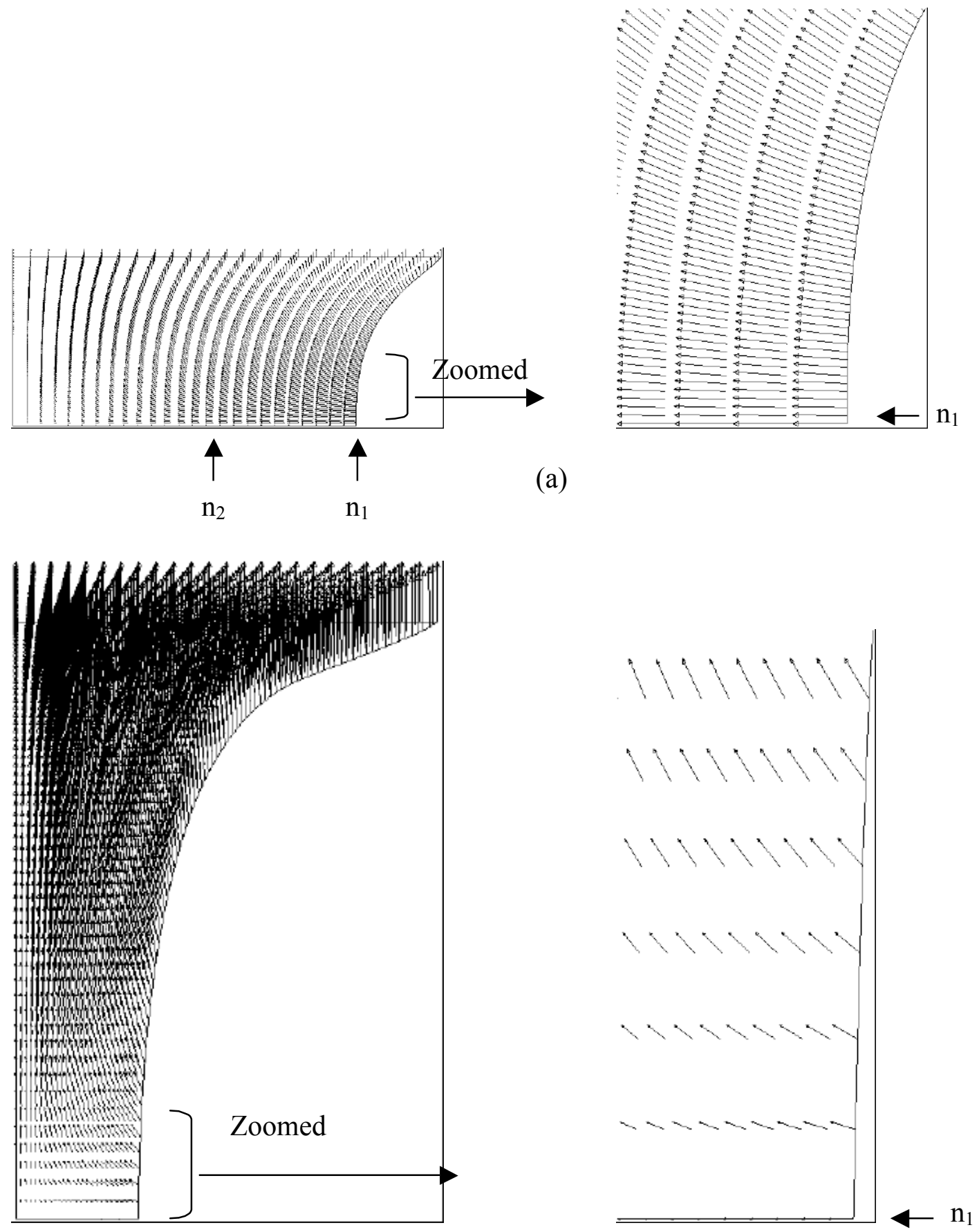

(b)

Figure 9. Velocity field: half-length model, (a) $\neg=0.32$, (b) $\square=1.6$, normal and zoomed views, Newtonian liquid-bridge. 


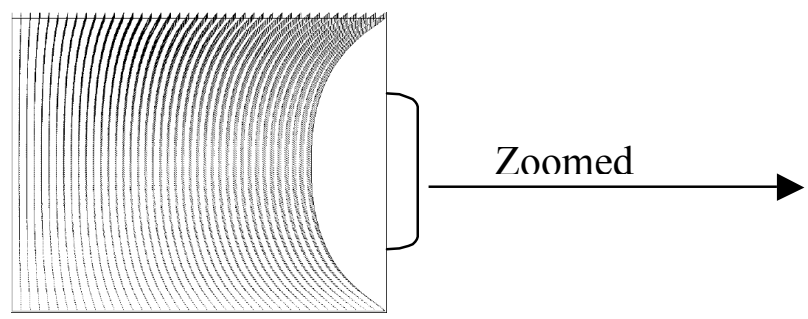

(a)

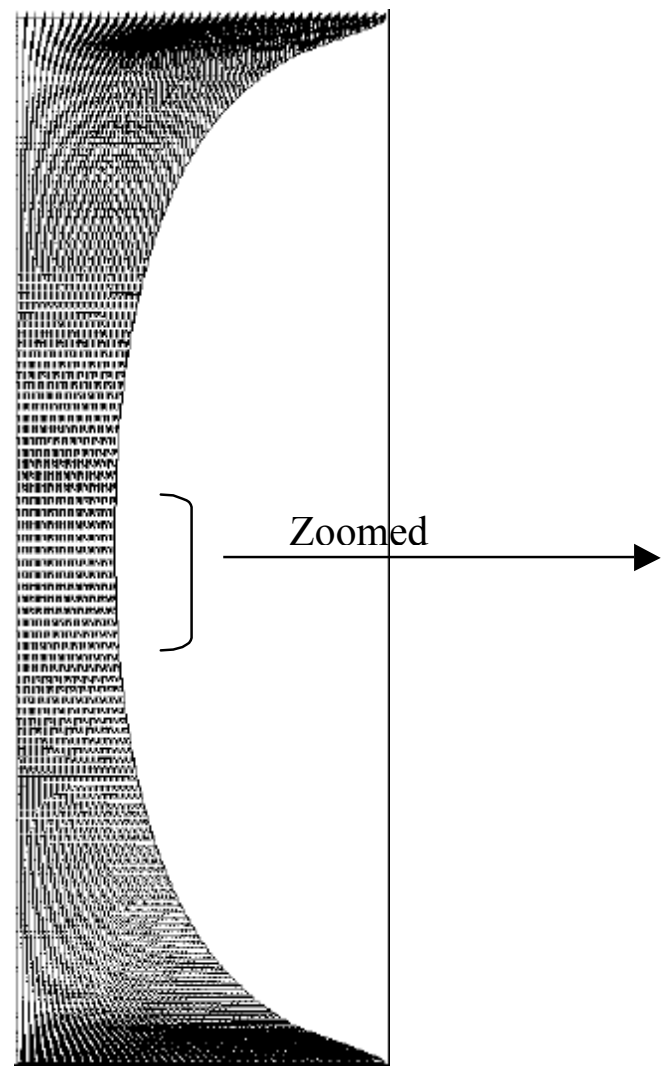

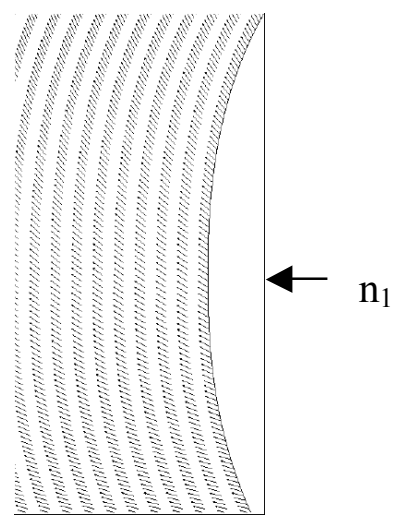

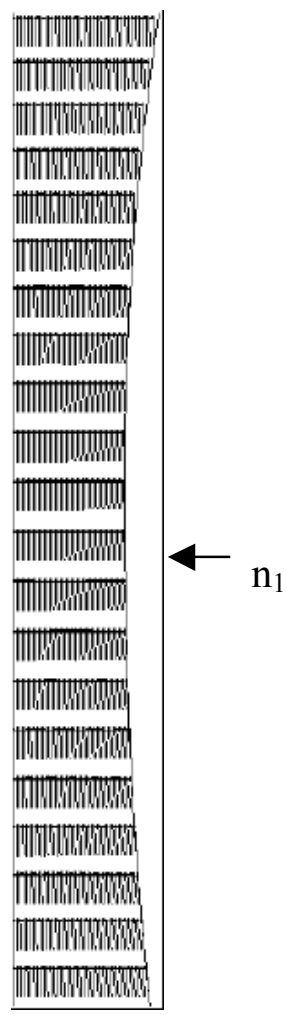

(b)

Figure 10. Velocity field: full-length model, (a) $\rceil=0.32$, (b) $\neg=1.6$, normal and zoomed views, Newtonian liquid-bridge. 


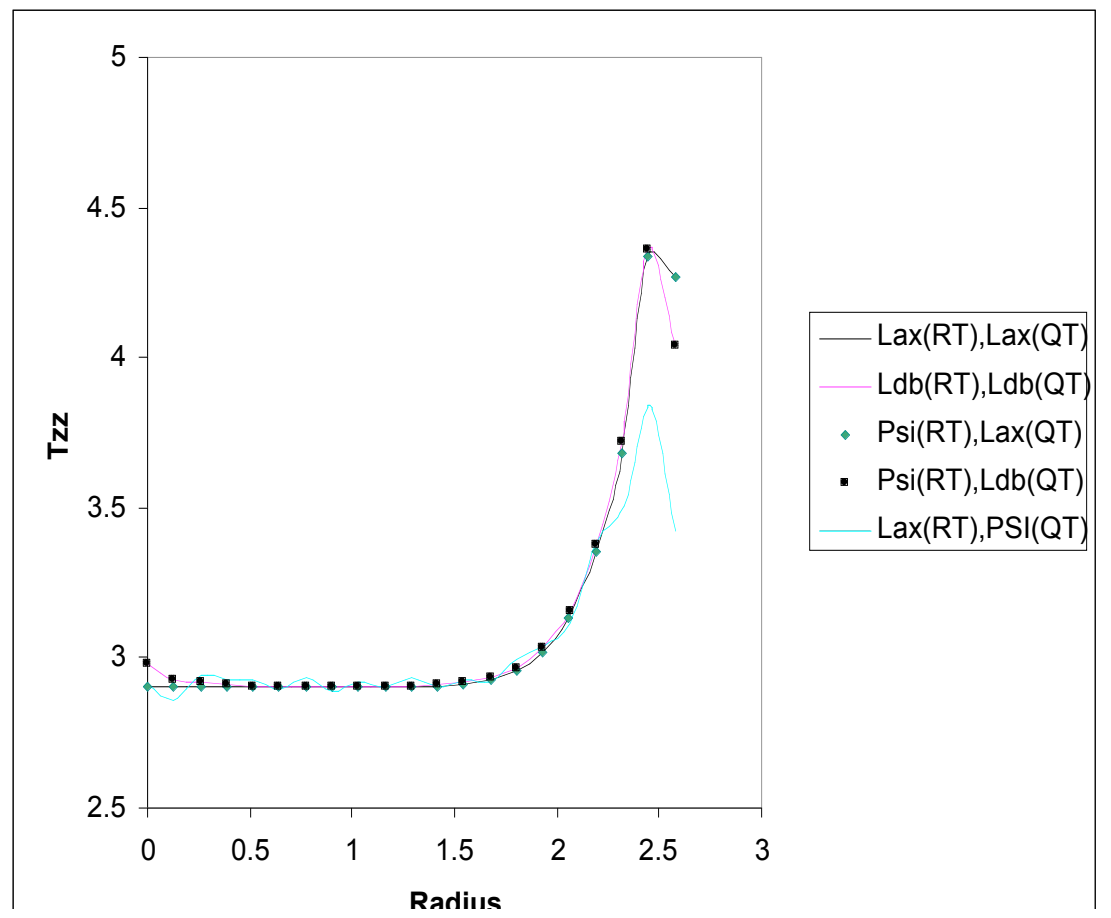

(a)

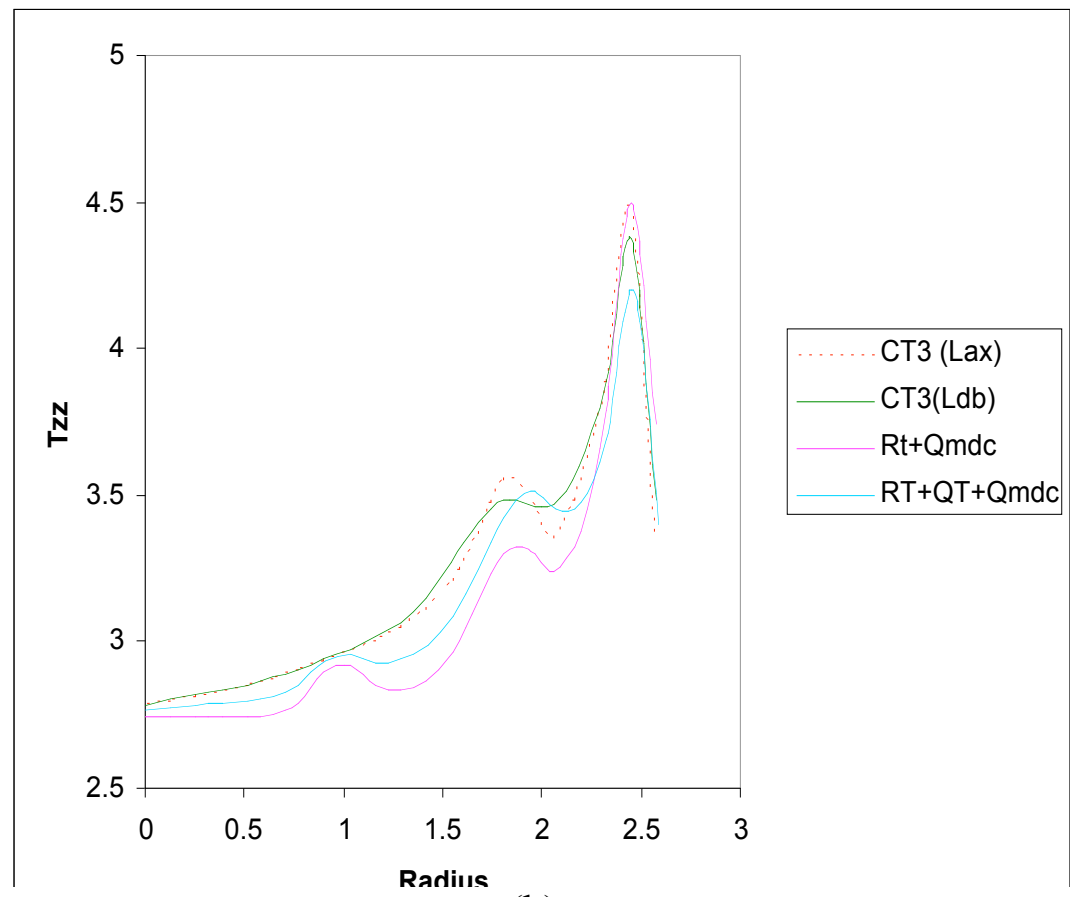

(b)

Figure 11. Total stress $\mathrm{T}_{\mathrm{zz}}$, along centre-line $\mathrm{Z}=\mathrm{L}(\mathrm{t}) / 2, W e=4$, initial aspect ratio $\square_{0}=\frac{1}{3}$ :

(a) different FD-schemes; (b) mdc-inclusion. 


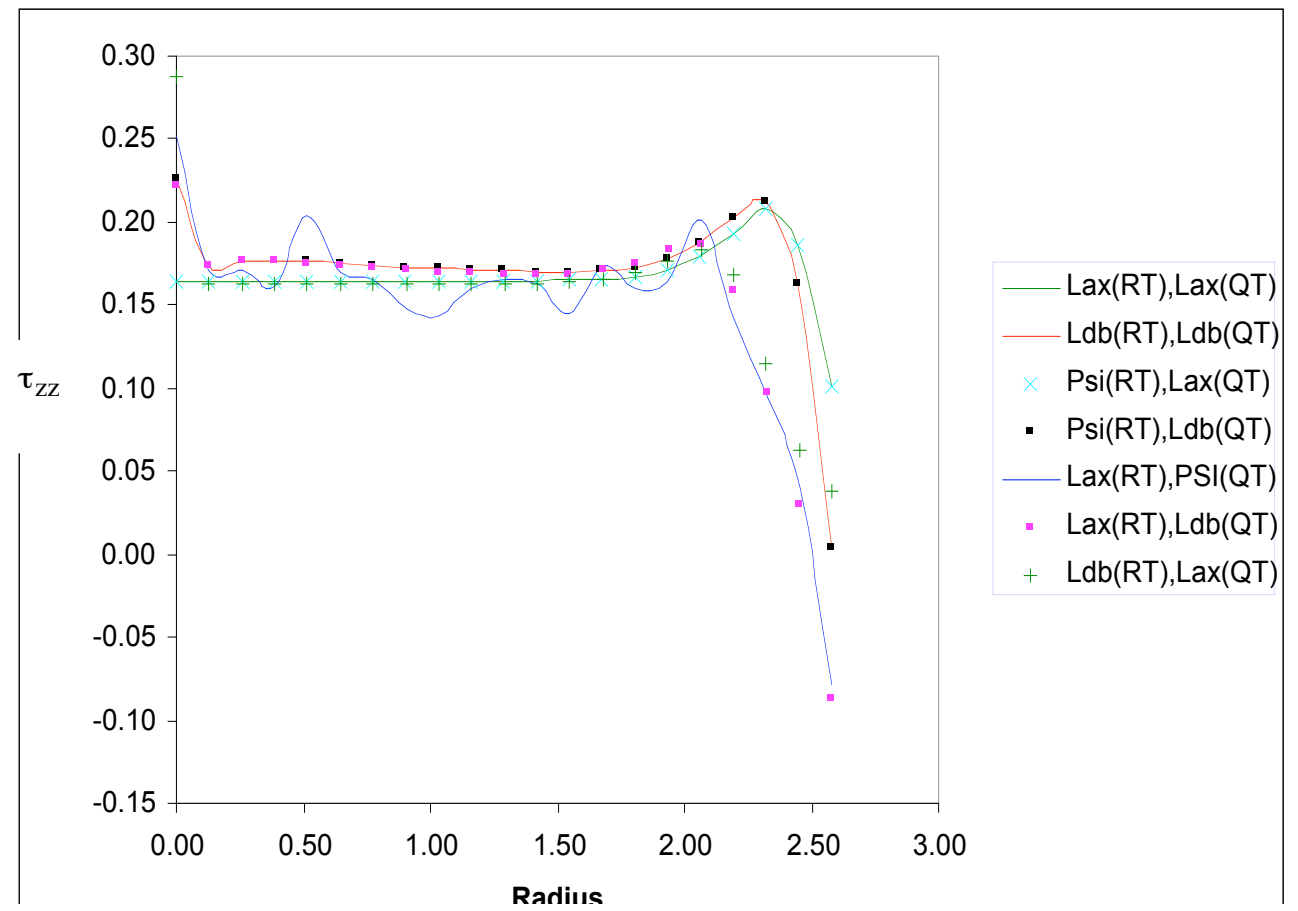

(a)

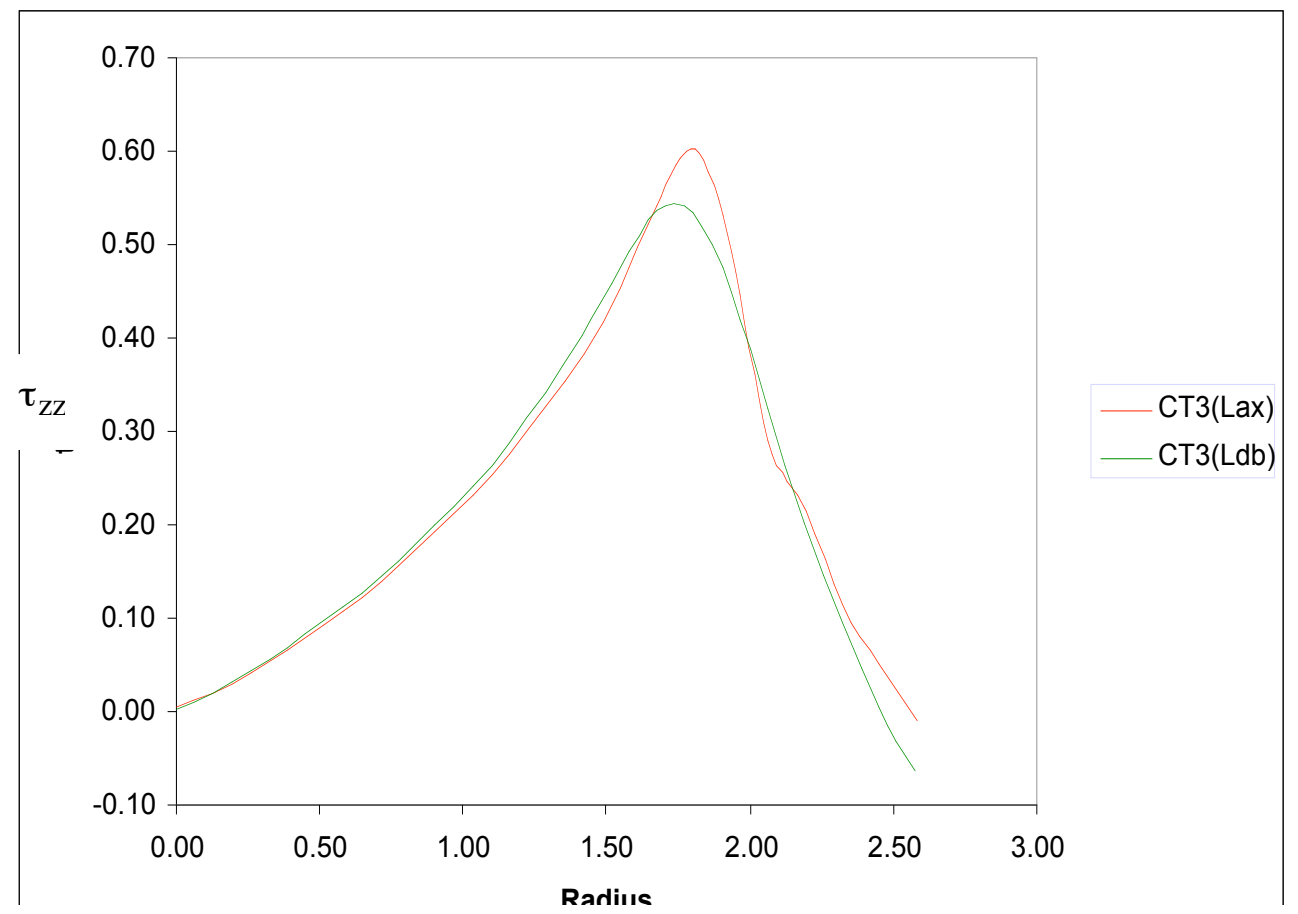

(b)

Figure 12. Extra-stress $\square_{\mathrm{zz}}$, along centre-line at $\mathrm{Z}=\mathrm{L}(\mathrm{t}) / 2, W e=4$, initial aspect ratio $\square_{0}=\frac{1}{3}$ :

(a) pure and hybrid fluctuation distribution FV-schemes; (b) mdc-inclusion. 

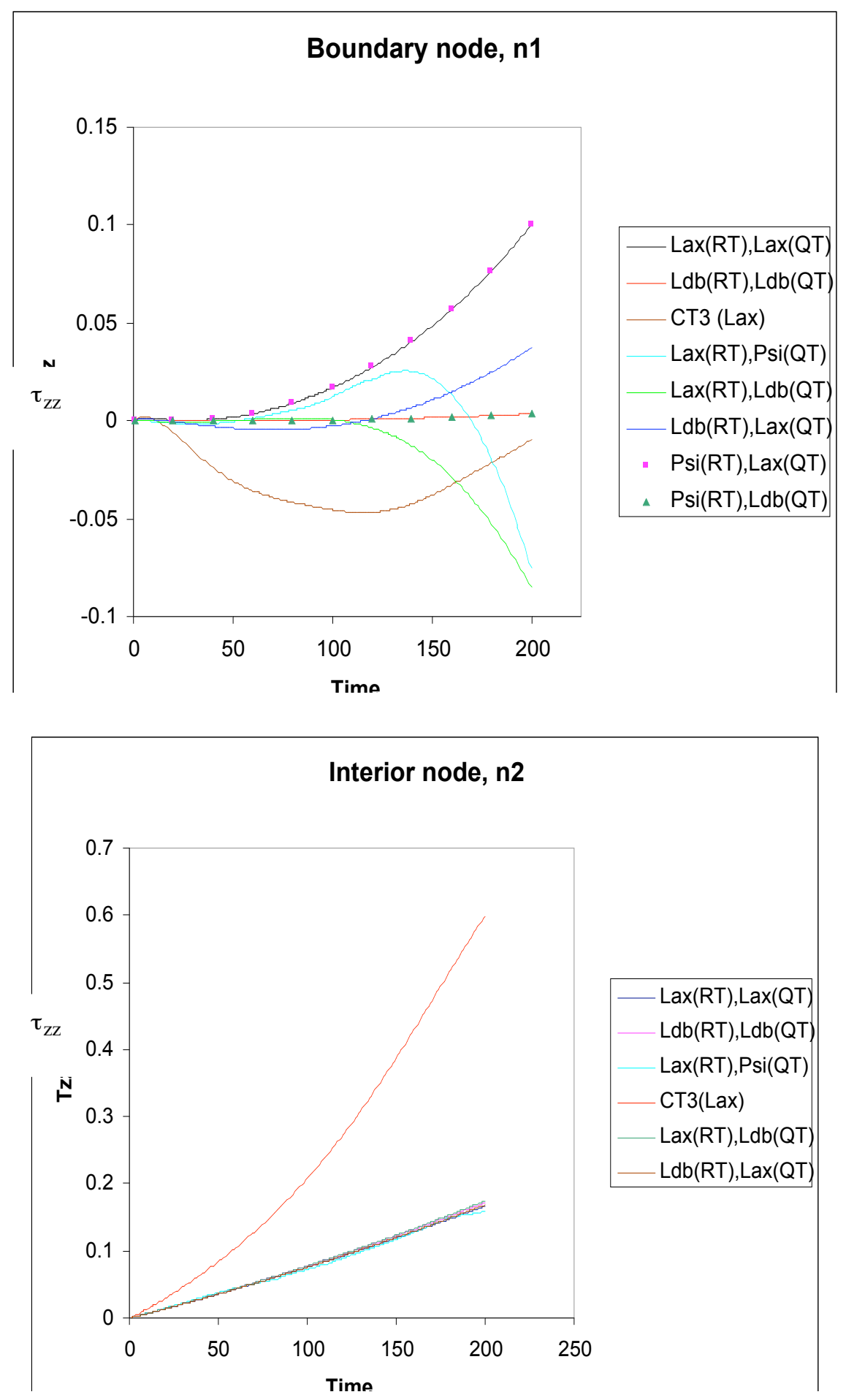

Figure 13. Transient development of extra-stress $\square_{z z}, \square=0$ to 0.2 , (a) node $n_{1}$, (b) node $n_{2}$ 


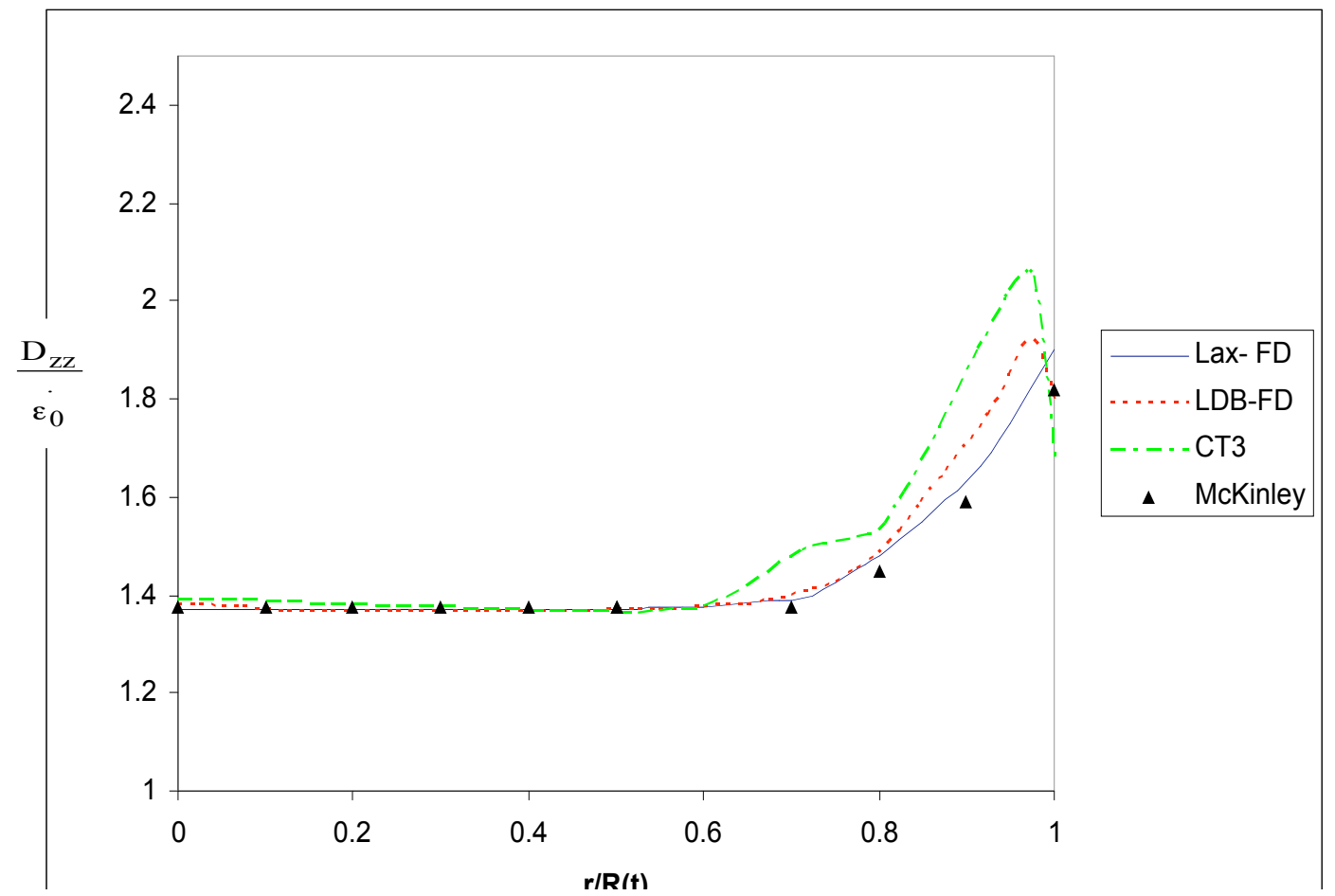

Figure. 14. Comparison of $\mathrm{D}_{\mathrm{zz}}$ along $\mathrm{Z}=\mathrm{L}(\mathrm{t}) / 2$, different schemes versus literature aspect $\mathrm{ratio}=1 / 3, \mathrm{De}=0.5$. 